\title{
The zero truncated Poisson Burr $X$ family of distributions with properties, characterizations, applications, and valida- tion test
}

T. H. M. Abouelmagd ${ }^{a, *}$, Mohammed S. Hamed ${ }^{a}$, G. G. Hamedani ${ }^{b}$, M. Masoom Alic, Hafida Goual ${ }^{d}$, Mustafa Ç. Korkmaz $^{\mathrm{e}}$, Haitham M. Yousof ${ }^{f}$

a Management Information System Department, Taibah University, Saudi Arabia.

${ }^{b}$ Department of Mathematics, Statistics and Computer Science, Marquette University, USA.

${ }^{c}$ Department of Mathematical Sciences, Ball State University, Muncie, USA.

${ }^{d}$ Laboratory of Probability and Statistics, University of Badji Mokhtar, Annaba, Algeria.

${ }^{e}$ Department of Measurement and Evaluation, Artvin Çoruh University, Artvin, TURKEY.

${ }^{f}$ Department of Statistics, Mathematics and Insurance, Benha University, Egypt.

\begin{abstract}
The goal of this work is to introduce a new family of continuous distributions with a strong physical applications. Some statistical properties are derived, and certain useful characterizations of the proposed family of distributions are presented. Five applications are provided to illustrate the importance of the new family. A modified goodness-of- fit test for the new family in complete data case are investigated via two examples. We propose, as a first step, the construction of Nikulin-Rao-Robson statistic based on chi-squared fit tests for the new family in the case of complete data. The new test is based on the Nikulin-RaoRobson statistic separately proposed by [M. S. Nikulin, Theory Probab. Appl., 18 (1974), 559-568] and [K. C. Rao, B. S. Robson, Comm. Statist., 3 (1974), 1139-1153]. As a second step, an application to real data has been proposed to show the applicability of the proposed test.
\end{abstract}

Keywords: Validation test, maximum likelihood estimation, generating function, moments, zero truncated Poisson. 2010 MSC: 60E05, 62F10

(C)2019 All rights reserved.

\section{Introduction and physical motivation}

In this paper we propose and study a new family of distributions using the zero truncated Poisson (ZTP) distribution with a strong physical motivation. Suppose that a system has $\mathrm{N}$ subsystems functioning independently at a given time where $\mathrm{N}$ has ZTP distribution with parameter $\lambda$. It is the conditional

\footnotetext{
*Corresponding author

Email addresses: tabouelmagd@taibahu.edu.sa (T. H. M. Abouelmagd), moswilem@gmail.com (Mohammed S. Hamed), gholamhoss.hamedani@marquette.edu (G. G. Hamedani), mali@bsu.edu (M. Masoom Ali), goual.hafida@gmail.com (Hafida Goual), mcagatay@artvin.edu.tr (Mustafa Ç. Korkmaz), haitham.yousof@f com.bu.edu.eg (Haitham M. Yousof)
}

doi: $10.22436 /$ jnsa.012.05.05

Received: 2018-09-14 Revised: 2018-10-05 Accepted: 2018-11-17 
probability distribution of a Poisson-distributed random variable (r.v.), given that the value of the r.v. is not zero. The probability mass function (PMF) of $\mathrm{N}$ is given by

$$
\operatorname{PMF}_{Z T P}^{(\lambda)}(N=n)=\left[\exp (-\lambda) \lambda^{n}\right] /\left.\{n ![-\exp (-\lambda)+1]\}\right|_{(n=1,2, \ldots)} .
$$

Note that for ZTP r.v., the expected value $\mathrm{E}(\mathrm{N} \mid \lambda)$ and variance $\operatorname{Var}(\mathrm{N} \mid \lambda)$ are, respectively, given by

$$
\mathbf{E}(\mathrm{N} \mid \lambda)=\lambda /[-\exp (-\lambda)+1], \quad \text { and } \quad \operatorname{Var}(\mathrm{N} \mid \lambda)=\frac{\lambda+\lambda^{2}}{[-\exp (-\lambda)+1]}-\frac{\lambda^{2}}{[-\exp (-\lambda)+1]^{2}}
$$

Suppose that the failure time of each subsystem has the Burr X generator ("BrX-G( $\theta, \varphi)$ " for short) defined by the cumulative distribution function (CDF) and probability density function (PDF) given by

$$
\mathrm{H}_{\mathrm{BrX}-\mathrm{G}}^{(\theta, \varphi)}(x)=\left\{1-\exp \left[-\left(\frac{\mathrm{G}^{(\varphi)}(x)}{\overline{\mathrm{G}}^{(\varphi)}(x)}\right)^{2}\right]\right\}^{\theta}, x \in \mathbb{R},
$$

and

$$
\begin{aligned}
h_{\mathrm{BrX}-\mathrm{G}}^{(\theta, \varphi)}(x)= & 2 \theta g^{(\varphi)}(x) \mathrm{G}^{(\varphi)}(x)\left[\overline{\mathrm{G}}^{(\varphi)}(x)\right]^{-3} \\
& \times \exp \left[-\left(\frac{\mathrm{G}^{(\varphi)}(x)}{\bar{G}^{(\varphi)}(x)}\right)^{2}\right]\left\{1-\exp \left[-\left(\frac{\mathrm{G}^{(\varphi)}(x)}{\bar{G}^{(\varphi)}(x)}\right)^{2}\right]\right\}^{\theta-1}, x \in \mathbb{R},
\end{aligned}
$$

respectively, where $\theta>0$ is a shape parameters, $\varphi$ is the parameter vector and $1-G^{(\varphi)}(\chi)=\bar{G}^{(\varphi)}(\chi)$. Let $Y_{i}$ denote the failure time of the ith subsystem and let $X=\min \left\{Y_{1}, Y_{2}, \cdots, Y_{N}\right\}$. Then the conditional CDF of $X$ given $N$ is

$$
\mathrm{F}(\mathrm{x} \mid \mathrm{N})=1-\operatorname{Pr}(\mathrm{X}>\mathrm{x} \mid \mathrm{N})=1-\left[1-\mathrm{H}_{\mathrm{BrX}-\mathrm{G}}^{(\theta, \varphi)}(\mathrm{x})\right]^{\mathrm{N}} .
$$

Therefore, the unconditional CDF of the ZTPBrX-G density function, can be expressed as

$$
\mathrm{F}_{\mathrm{ZTPBrX}-\mathrm{G}}^{(\lambda, \theta, \varphi)}(x)=[-\exp (-\lambda)+1]^{-1}\left[1-\exp \left(-\lambda\left\{1-\exp \left[-\left(\frac{\mathrm{G}^{(\varphi)}(\chi)}{\bar{G}^{(\varphi)}(\chi)}\right)^{2}\right]\right\}^{\theta}\right)\right]
$$

with the corresponding PDF as

$$
\begin{aligned}
f_{Z T P B r X-G}^{(\lambda, \theta, \varphi)}(x)= & 2 \theta \lambda[-\exp (-\lambda)+1]^{-1} g^{(\varphi)}(x) G^{(\varphi)}(x)\left[\bar{G}^{(\varphi)}(x)\right]^{-3} \\
& \times \exp \left(-\lambda\left\{1-\exp \left[-\left(\frac{G^{(\varphi)}(x)}{\bar{G}^{(\varphi)}(x)}\right)^{2}\right]\right\}^{\theta}\right) \\
& \times \exp \left[-\left(\frac{G^{(\varphi)}(x)}{\bar{G}^{(\varphi)}(x)}\right)^{2}\right]\left\{1-\exp \left[-\left(\frac{G^{(\varphi)}(x)}{\bar{G}^{(\varphi)}(x)}\right)^{2}\right]\right\}^{\theta-1} .
\end{aligned}
$$

Some useful new families can be cited as mentioned in the parentheses (see $[8,9,13,18,19,23,27,38-41]$ ). It is not shown Which of them is this. among others).

This article is organized as follows. Some special submodels are presented in Section 2. In Section 3, we derive some of the mathematical properties of the new family. Some useful Characterizations results are presented in Section 4. Maximum likelihood estimation for the model parameters under uncensored data is addressed in Section 5. In Sections 6 and 7, the potentiality of the proposed family is illustrated by means of five real data sets. A modified goodness-of-fit test for the new family in complete data case is presented in Section 8. Finally, Section 9 provides some concluding remarks. 


\section{Special ZTPBrX-G submodels}

\subsection{The ZTPBrX-Weibull (ZTPBrXW) distribution}

Consider the CDF and PDF (for $x>0$ )

$$
\mathrm{G}_{W}^{(\alpha, \beta)}(x)=\left\{1-\exp \left[-(\alpha x)^{\beta}\right]\right\} \text { and } g_{W}^{(\alpha, \beta)}(x)=\beta \alpha^{\beta} x^{\beta-1} \exp \left[-(\alpha x)^{\beta}\right],
$$

respectively, of the Weibull distribution with positive parameters $\alpha$ and $\beta$. Then, the PDF of the ZTPBrXW model will be obtained directly via insrting $G_{W}^{(\alpha, \beta)}(x)$ and $g_{W}^{(\alpha, \beta)}(x)$ into (1.1) and (1.2) to obtain

$$
\begin{aligned}
f_{\mathrm{ZTPBrXW}}^{(\lambda, \theta, \alpha, \beta)}(x)= & 2 \theta \lambda \beta \alpha^{\beta} \chi^{\beta-1}[-\exp (-\lambda)+1]^{-1} \exp \left[2(\alpha x)^{\beta}\right] \\
& \times\left\{1-\exp \left[-(\alpha x)^{\beta}\right]\right\} \exp \left(-\lambda\left\{1-\exp \left[-\left\{\exp \left[(\alpha x)^{\beta}\right]\right\}-1\right]\right\}^{\theta}\right) \\
& \times \exp \left[-\left\{\exp \left[(\alpha x)^{\beta}\right]\right\}-1\right]\left\{1-\exp \left[-\left\{\exp \left[(\alpha x)^{\beta}\right]\right\}-1\right]\right\}^{\theta-1} .
\end{aligned}
$$

The ZTPBrXW distribution includes the ZTPBrX-Rayleigh (ZTPBrXR) distribution when $\beta=2$. For $\beta=1$, we have the ZTPBrX-exponential (ZTPBrXE) distribution. The plots of the ZTPBrXW density for selected parameter values are displayed in Figure 1. From Figure 1 we note that the ZTPBrXW model will be suitable for modeling symmetric, right skewed, left skewed and unimodal data sets. The HRF of the ZTPBrXW may have the increasing, decreasing and bathtub shapes.
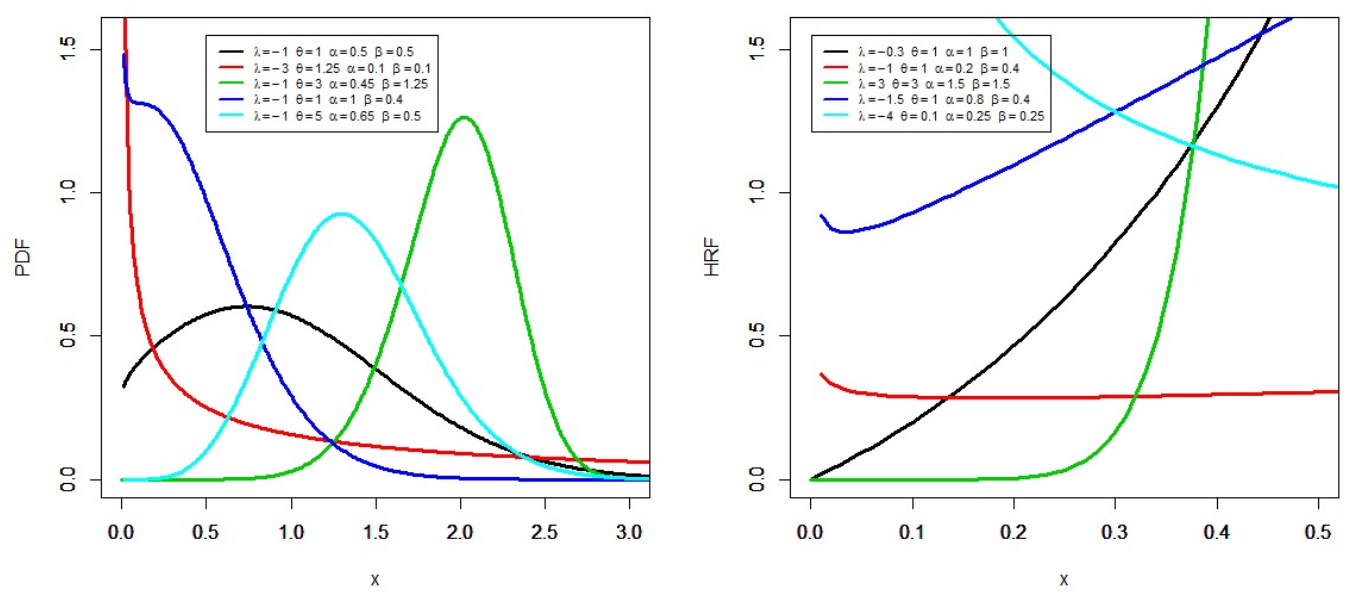

Figure 1: PDF and HRF plots for the ZTPBrXW model.

\subsection{The $\mathrm{ZTPBrX}$-Lomax (ZTPBrXLx) distribution}

The CDF and PDF (for $x>0$ ) of the Lx distribution with positive parameters $\alpha$ and $\beta$ are

$$
\mathrm{G}_{\mathrm{Lx}}^{(\alpha, \beta)}(x)=1-\left[1+\left(x \beta^{-1}\right)\right]^{-\alpha} \text { and } \mathrm{g}_{\mathrm{Lx}}^{(\alpha, \beta)}(x)=\alpha \beta^{-1}\left[1+\left(x \beta^{-1}\right)\right]^{-\alpha-1},
$$

respectively. Then, the PDF of the ZTPBrXLx model will be obtained directly via inserting $G_{L x}^{(\alpha, \beta)}(x)$ and $\mathrm{g}_{\mathrm{Lx}}^{(\alpha, \beta)}(\chi)$ into (1.1) and (1.2) to obtain

$$
f_{\mathrm{ZTPBrXLx}}^{(\lambda, \theta, \alpha, \beta)}(x)=2 \theta \lambda \alpha \beta^{-1}[-\exp (-\lambda)+1]^{-1}\left[1+\left(x \beta^{-1}\right)\right]^{2 \alpha-1}
$$




$$
\begin{aligned}
& \times\left[1-\exp \left(-\left\{\left[1+\left(x \beta^{-1}\right)\right]^{\alpha}-1\right\}^{2}\right)\right]^{\theta-1} \\
& \times \exp \left(-\lambda\left\{1-\exp \left(-\left\{\left[1+\left(x \beta^{-1}\right)\right]^{\alpha}-1\right\}^{2}\right)\right\}^{\theta}\right) \\
& \times\left\{1-\left[1+\left(x \beta^{-1}\right)\right]^{-\alpha}\right\} \exp \left(-\left\{\left[1+\left(x \beta^{-1}\right)\right]^{\alpha}-1\right\}^{2}\right) .
\end{aligned}
$$

The plots of the ZTPBrXLx density are displayed in Figure 2 for selected parameter values. From Figure 2 , we conclude that the ZTPBrXLx model will be suitable for various shaped data sets. The HRF of the ZTPBrXW may have the decreasing, increasing, and unimodal shapes.
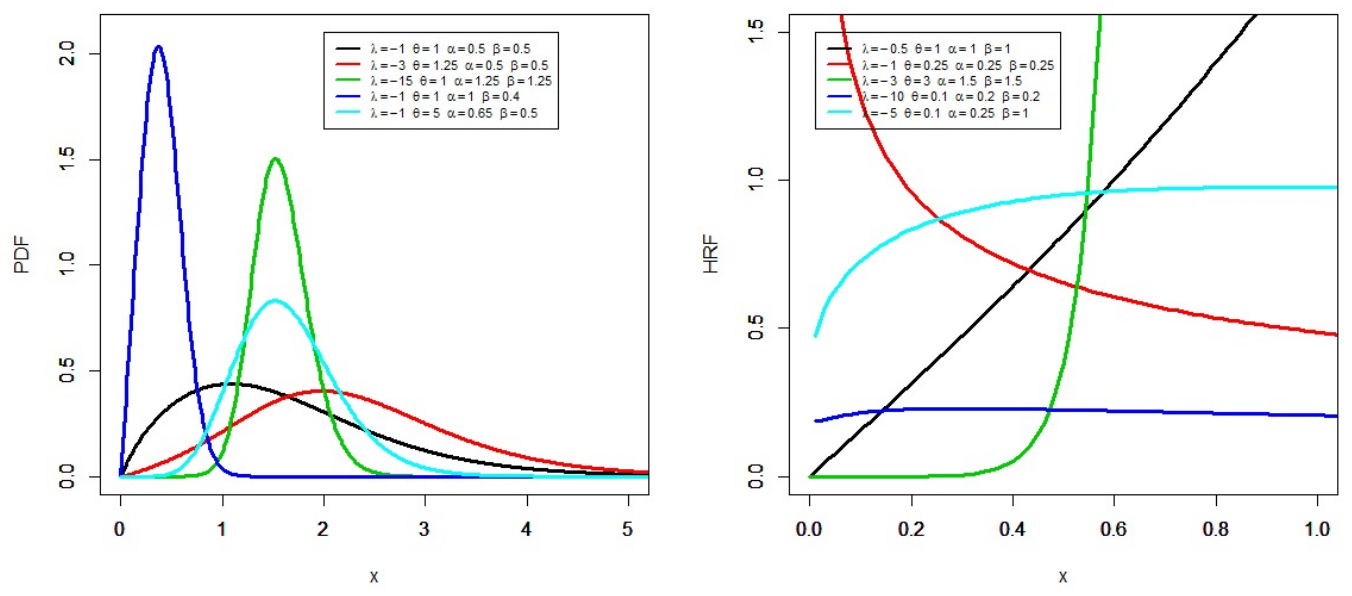

Figure 2: PDF and HRF plots for the ZTPBrXLx model.

\section{Mathematical properties}

\subsection{Useful expansions}

Using the power series

$$
\exp (\zeta)=\sum_{\mathrm{q}=0}^{\infty} \frac{\zeta^{\mathrm{q}}}{\mathrm{q} !}, \zeta \in \Re,
$$

the PDF in (1.2) can be written as

$$
\begin{aligned}
f_{Z \operatorname{TPBrX}-\mathrm{G}}^{(\lambda, \theta, \varphi)}(x)= & \sum_{h=0}^{\infty} \frac{2 \theta \lambda^{1+h}(-1)^{h}}{\mathrm{~h} ![-\exp (-\lambda)+1]} \exp \left[-\left(\frac{\mathrm{G}^{(\varphi)}(x)}{\overline{\mathrm{G}}^{(\prime)}(x)}\right)^{2}\right] \\
& \times \frac{g^{(\varphi)}(x) \mathrm{G}^{(\prime)}(x)}{\left[\overline{\mathrm{G}}^{(\varphi)}(x)\right]^{3}}\left\{1-\exp \left[-\left(\frac{\mathrm{G}^{(\varphi)}(x)}{\overline{\mathrm{G}}^{(\prime)}(x)}\right)^{2}\right]\right\}^{\theta(h+1)-1} .
\end{aligned}
$$

If $|s|<1$ and $c>0$ is a real non-integer, the following power series holds

$$
(1-s)^{c-1}=\sum_{i=0}^{\infty} \frac{(-1)^{i} \Gamma(c)}{i ! \Gamma(c-i)} s^{i} .
$$


Applying (3.2) to (3.1) we have

$$
\begin{aligned}
f_{Z \operatorname{TPBrX}-\mathrm{G}}^{(\lambda, \theta, \varphi)}(x)= & \frac{2 \theta g^{(\varphi)}(x) \mathrm{G}^{\left({ }^{\prime}\right)}(x)}{[-\exp (-\lambda)+1]}\left[\overline{\mathrm{G}}^{(\varphi)}(x)\right]^{-3} \\
& \times \sum_{h, i=0}^{\infty} \frac{\lambda^{1+h}(-1)^{h+i} \Gamma(\theta(h+1))}{i ! \Gamma(\theta(h+1)-i)} \exp \left[-(i+1)\left(\frac{\mathrm{G}^{(\varphi)}(x)}{\bar{G}^{(\varphi)}(x)}\right)^{2}\right] .
\end{aligned}
$$

Applying the power series to the term $\exp \left[-(i+1)\left(G^{(\varphi)}(x) / \bar{G}^{(\varphi)}(x)\right)^{2}\right]$, equation (3.3) becomes

$$
f_{\mathrm{ZTPBrX}-\mathrm{G}}^{(\lambda, \theta, \varphi)}(x)=\sum_{h, i, j=0}^{\infty} \frac{2 \theta \lambda^{1+h}(-1)^{h+i+j}(i+1)^{j} \Gamma(\theta(h+1))}{i ! j !\left(1-e^{-\lambda}\right) \Gamma(\theta(h+1)-i)} \frac{g^{(\varphi)}(x) G^{(\varphi)}(x)^{2 j+1}}{\left[\bar{G}^{(\varphi)}(x)\right]^{2 j+3}} .
$$

Consider the series expansion

$$
\left.(1-\zeta)^{-c}\right|_{(|\zeta|<1, c>0)}=\sum_{k=0}^{\infty} \frac{\Gamma(c+k)}{k ! \Gamma(c)} \zeta^{k}
$$

Applying the expansion in (3.5) to (3.4) for the term $\left[\overline{\mathrm{G}}^{(\varphi)}(x)\right]^{2 j+3}$, equation (3.4) becomes

$$
\begin{aligned}
f_{Z T P B r X-G}^{(\lambda, \theta, \varphi)}(x)= & \sum_{h, i, j, k=0}^{\infty} \frac{2 \theta \lambda^{1+h}(-1)^{h+i+j}(i+1)^{j}[2(1+j)+k]}{i ! j ! k ![-\exp (-\lambda)+1][2(1+j)+k]} \\
& \times \frac{\Gamma(\theta(h+1)) \Gamma(3+2 j+k)}{\Gamma(\theta(h+1)-i) \Gamma(2 j+3)} g^{(\varphi)}(x) G^{\left({ }^{\prime}\right)}(x)^{2 j+k+1} .
\end{aligned}
$$

This can be written as

$$
f_{\mathrm{ZTPBrX}-\mathrm{G}}^{(\lambda, \theta, \varphi)}(x)=\sum_{j, k=0}^{\infty} v_{j, k} \pi_{2(1+j)+k}(x ; \varphi),
$$

where

$$
v_{j, k}=\frac{2 \theta \lambda^{1+h}(-1)^{j} \Gamma(3+2 j+k)}{j ! k ![-\exp (-\lambda)+1] \Gamma(2 j+3)[2(1+j)+k]} \sum_{h, i=0}^{\infty} \frac{(-1)^{h+i} \Gamma(\theta(h+1))(i+1)^{j}}{i ! \Gamma(\theta(h+1)-i)},
$$

and

$$
\pi_{2(1+j)+k}(x ; \varphi)=[2(1+j)+k] g^{(\varphi)}(x)\left[G^{\left({ }^{\prime}\right)}(x)\right]^{2 j+k+1},
$$

which is the PDF of the exp-G family with power parameter $2(1+j)+k$. Equation (3.6) reveals that the density of $X$ can be expressed as a linear mixture of exp-G densities. So, several mathematical properties of the new family can be obtained from those of the exp-G distribution. Similarly, the CDF of the ZTPBrX-G family can also be expressed as a mixture of exp-G CDFs given by

$$
\mathrm{F}_{\mathrm{ZTPBrX}-\mathrm{G}}^{(\lambda, \theta, \varphi)}(x)=\sum_{j, k=0}^{\infty} v_{j, k} \Pi_{2(1+j)+k}(x ; \varphi),
$$

where $\Pi_{2(1+j)+k}(x ; \varphi)$ is the CDF of the exp-G family with power parameter $2(1+j)+k$.

3.2. Quantile and random number generation

The quantile function (QF) of $X$, where $X \sim \operatorname{ZTPBrX}-G(\lambda, \theta, \varphi)$, is obtained by inverting (1.1) as

$$
Q(u)=G^{-1}\left(1+\left\{-\ln \left[1-\left(\frac{-\ln \{1-u[-\exp (-\lambda)+1]\}}{\lambda}\right)^{\frac{1}{\theta}}\right]\right\}^{\frac{1}{2}}\right), 0 \leqslant u \geqslant 1 .
$$

Simulating the ZTPBrX-G r.v. is straightforward. If $U$ is a uniform variate on the unit interval $(0,1)$, then 
the r.v. $X=Q(U)$ follows (1.2).

\subsection{Quantile spread ordering}

The quantile spread (QS) of the r.v. Z Z ZTPBrX-G $(\lambda, \theta, \varphi)$ having CDF (1.1) is given by

$$
\mathrm{QS}_{X}(\mathbf{1})=\left.\left\{\left[\mathrm{F}^{-1}(\mathbf{1})\right]-\left[\mathrm{F}^{-1}(1-\mathbf{1})\right]\right\}\right|_{(\mathbf{1} \in(0.5,1))},
$$

which also implies that

$$
\mathrm{QS}_{\mathrm{X}}(\mathbf{1})=\left[\mathrm{S}^{-1}(1-\mathbf{1})\right]-\left[\mathrm{S}^{-1}(\mathbf{1})\right]
$$

where

$$
\mathrm{F}^{-1}(\mathbf{1})=\mathrm{S}^{-1}(1-\mathbf{1}) \text { and } \mathrm{S}(z)=1-\mathrm{F}(z),
$$

is the survival function (SF). The QS of a distribution describes how the probability mass is placed symmetrically about its median and hence can be used to formalize concepts such as peakedness and tail weight traditionally associated with kurtosis. So, it allows us to separate concepts of kurtosis and peakedness for asymmetric models. Let $Z_{1}$ and $Z_{2}$ be two random variables following the ZTPBrX-G $(\lambda, \theta, \varphi)$ with quantile spreads $Q S_{Z_{1}}$ and $Q S_{Z_{2}}$, respectively. Then $Z_{1}$ is called smaller than $Z_{2}$ in quantile spread order, denoted by $Z_{1} \leqslant Q s Z_{2}$, if

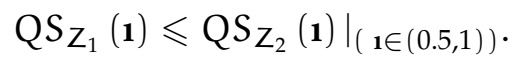

The following properties of the QS order can be obtained:

1. if $Z_{1}$ and $Z_{2}$ have the same distribution then

$$
\mathrm{QS}_{\mathrm{Z}_{1}}=\mathrm{QS}_{\mathrm{Z}_{2}}
$$

2. the order $\leqslant \mathrm{Q} S$ is location-free

$$
\mathrm{Z}_{1} \leqslant \mathrm{QS} \mathrm{Z}_{2} \text { if }\left.\left[\left(\mathrm{Z}_{1}+\mathrm{C}\right) \leqslant \mathrm{QS} \mathrm{Z}_{2}\right]\right|_{\left(\mathrm{C} \in \mathbb{R}^{+}\right)}
$$

3. the order $\leqslant \mathrm{Q} S$ is dilative

$$
\mathrm{Z}_{1} \leqslant \mathrm{QS} C \mathrm{Z}_{1} \text { whenever } \mathrm{C} \geqslant 1 \text { and } \mathrm{Z}_{2} \leqslant\left.\mathrm{QS} \quad C Z_{2}\right|_{(\mathrm{C} \geqslant 1)} ;
$$

4. let $F_{Z_{1}}$ and $F_{Z_{2}}$ be symmetric, then

$$
\mathrm{Z}_{1} \leqslant \mathrm{QS} \mathrm{Z}_{2} \text { if and only if } \mathrm{F}_{\mathrm{Z}_{1}}^{-1}(\mathbf{1}) \leqslant\left.\mathrm{F}_{\mathrm{Z}_{2}}^{-1}(\mathbf{1})\right|_{(\mathbf{1} \in(0.5,1))} ;
$$

5. the order $\leqslant \mathrm{QS}$ implies ordering of the mean absolute deviation around the median, say $\left.\operatorname{MAD}\left(Z_{i}\right)\right|_{(i=1.2)}$,

$$
\mathbf{E}\left[\left|Z_{1}-\operatorname{Median}\left(Z_{1}\right)\right|\right]=\operatorname{MAD}\left(Z_{1}\right)
$$

and

$$
\mathbf{E}\left[\left|Z_{2}-\operatorname{Median}\left(Z_{2}\right)\right|\right]=\operatorname{MAD}\left(Z_{2}\right),
$$

where

$$
\mathrm{Z}_{1} \leqslant \mathrm{QS} \mathrm{Z}_{2} \Rightarrow \operatorname{MAD}\left(\mathrm{Z}_{1}\right) \leqslant \mathrm{QS} \operatorname{MAD}\left(\mathrm{Z}_{2}\right)
$$

Finally

$$
\mathrm{Z}_{1} \leqslant \mathrm{QS} \mathrm{Z}_{2} \text { if and only if }-\mathrm{Z}_{1} \leqslant \mathrm{QS}-\mathrm{Z}_{2}
$$

\subsection{Moments}

Let $Y_{2(1+j)+k}$ be a r.v. having density $\pi_{2(1+j)+k}(x ; \varphi)$. The rth ordinary moment of $X$, say $\mu_{r}^{\prime}$, follows from (3.6) as

$$
\mu_{r}^{\prime}=\mathbf{E}\left(X^{r}\right)=\sum_{j, k=0}^{\infty} v_{j, k} E\left(Y_{2(1+j)+k}^{r}\right),
$$


where

$$
\mathbf{E}\left(\mathrm{Y}_{\zeta}^{\mathrm{r}}\right)=\zeta \int_{-\infty}^{\infty} \chi^{\mathrm{r}} \mathrm{g}^{(\varphi)}(\chi) \mathrm{G}^{(\varphi)}(\chi)^{\zeta-1} \mathrm{~d} x,
$$

can be evaluated numerically in terms of the baseline qf $\mathrm{Q}_{\mathrm{G}}(\mathrm{u})=\mathrm{G}^{-1}(\mathrm{u})$ as

$$
\mathbf{E}\left(\mathrm{Y}_{\zeta}^{\mathrm{r}}\right)=\zeta \int_{0}^{1} \mathrm{u}^{\zeta-1}\left[\mathrm{Q}_{\mathrm{G}}(\mathrm{u})\right]^{\mathrm{r}} \mathrm{du} \text {. }
$$

Setting $r=1$ in (3.7) gives the mean of X. For the ZTPBrXW model we have

$$
\mu_{r}^{\prime}=\mathbf{E}\left(X^{r}\right)=\sum_{j, k=0}^{\infty} v_{j, k} \mathbf{E}\left(Y_{2(1+j)+k}^{r}\right)=\left.\sum_{j, k, h=0}^{\infty} \tau_{j, k, h}^{\{[2(1+j)+k], r\}} \alpha^{r} \Gamma\left(1+r \beta^{-1}\right)\right|_{(r>-\beta)},
$$

where

$$
\tau_{j, k, h}^{\{[2(1+j)+k], r\}}=v_{j, k} \tau_{h}^{\{[2(1+j)+k], r\}},
$$

and

$$
\tau_{m}^{\{1+\zeta, s\}}=\frac{(1+\zeta)(-1)^{m}}{(1+m)^{\left(1+\frac{s}{\beta}\right)}}\left(\begin{array}{c}
\zeta \\
m
\end{array}\right) .
$$

\subsection{Incomplete moments}

The $r$ th incomplete moment of $X$ is defined by $m_{r}(y)=\int_{-\infty}^{y} x^{r} f(x) d x$. We can write from (3.6)

$$
m_{r}(y)=\sum_{j, k=0}^{\infty} v_{j, k} m_{r, 2(1+j)+k}(y)
$$

where

$$
m_{r, \alpha}(y)=\int_{0}^{G(y)} u^{\alpha-1}\left[Q_{G}(u)\right]^{r} d u
$$

The integral $m_{r, \alpha}(y)$ can be determined analytically for special models with closed-form expressions for $\mathrm{Q}_{\mathrm{G}}(\mathrm{u})$ or computed at least numerically for most baseline distributions. Two important applications of the first incomplete moment are related to the mean deviations about the mean and median and to the Bonferroni and Lorenz curves. For the ZTPBrXW model we have

$$
m_{r}(y)=\left.\sum_{j, k, h=0}^{\infty} \tau_{j, k, h}^{\{[2(1+j)+k], r\}} \alpha^{r} \gamma\left(1+r \beta^{-1},\left(\frac{a}{t}\right)^{\beta}\right)\right|_{(r>-\beta)}
$$

where

$$
\gamma(a, q)=\int_{0}^{q} t^{a-1} \exp (-t) d t=\sum_{h=0}^{\infty} \frac{(-1)^{h}}{n !(a+h)} z^{a+h}
$$

\subsection{Generating functions}

The moment generating function (mgf) of $X$, say $M(t)=E(\exp (t X))$, is obtained from (3.6) as

$$
M(t)=\sum_{j, k=0}^{\infty} v_{j, k} M_{2(1+j)+k}(t),
$$

where $M_{\zeta}(t)$ is the generating function of $Y_{\zeta}$ given by

$$
M_{\zeta}(t)=\zeta \int_{-\infty}^{\infty} \exp (t x) g^{(\varphi)}(x)\left[G^{(\varphi)}(x)\right]^{\zeta-1} d x=\zeta \int_{0}^{1} \exp \left[t Q_{G}(u ; \alpha)\right] u^{\zeta-1} d u .
$$


The last two integrals can be computed numerically for most parent distributions. For the ZTPBrXW model we have

$$
M(t)=\left.\sum_{j, k, h, r}^{\infty} \tau_{j, k, h, r}^{\{[2(1+j)+k], r\}} \alpha^{r} \Gamma\left(1+r \beta^{-1}\right)\right|_{(r>-\beta)}
$$

where

$$
\tau_{j, k, h, r}^{\{[2(1+j)+k], r\}}=\frac{t^{r}}{r !} \tau_{j, k, h}^{\{[2(1+j)+k], r\}} .
$$

\section{Characterizations results}

This section deals with the characterizations of the ZTPBrX-G distribution based on the ratio of two truncated moments. Note that our characterizations can be employed also when the cdf does not have a closed form. We would also like to mention that due to the nature of ZTPBrX-G distribution, our characterizations may be the only possible ones. Our first characterization employs a theorem due to [15], see Theorem .1 of Appendix. The result, however, holds also when the interval $\mathrm{H}$ is not closed, since the condition of the Theorem is on the interior of $\mathrm{H}$.

Proposition 4.1. Let $X: \Omega \rightarrow \mathbb{R}$ be a continuous random variable and let

$$
\mathrm{q}_{1}(x)=\left[\overline{\mathrm{G}}^{(\varphi)}(x)\right]^{3}\left\{1-\exp \left[-\left(\frac{\mathrm{G}^{(\varphi)}(x)}{\overline{\mathrm{G}}^{\left({ }^{\prime}\right)}(x)}\right)^{2}\right]\right\}^{1-\theta} \exp \left(\lambda\left\{1-\exp \left[-\left(\frac{\mathrm{G}^{(\prime)}(x)}{\overline{\mathrm{G}}^{(\varphi)}(x)}\right)^{2}\right]\right\}^{\theta}\right)
$$

and

$$
\mathrm{q}_{2}(x)=\mathrm{q}_{1}(x)\left[\mathrm{G}^{(\varphi)}(x)\right]^{2} \text { for } x \in \mathbb{R} .
$$

The random variable $X$ has PDF (1.2) if and only if the function $\eta$ defined in Theorem. 1 is of the form

$$
\eta(x)=\frac{1}{2}\left\{1+\left[G^{(\varphi)}(x)\right]^{2}\right\}, \quad x \in \mathbb{R} .
$$

Proof. Suppose the random variable $X$ has PDF (6), then

$$
(1-F(x)) E\left[q_{1}(X) \mid X \geqslant x\right]=\frac{\theta \lambda}{[1-\exp (-\lambda)]}\left\{1-\left[G^{(\varphi)}(x)\right]^{2}\right\}, \quad x \in \mathbb{R},
$$

and

$$
(1-F(x)) E\left[q_{2}(X) \mid X \geqslant x\right]=\frac{\theta \lambda}{2[1-\exp (-\lambda)]}\left\{1-\left[G^{(\varphi)}(x)\right]^{4}\right\}, \quad x \in \mathbb{R}
$$

Further,

$$
\eta(x) q_{1}(x)-q_{2}(x)=\frac{q_{1}(x)}{2}\left\{1-\left[G^{(\varphi)}(x)\right]^{2}\right\}>0, \text { for } x \in \mathbb{R} .
$$

Conversely, if $\eta$ is of the above form, then

$$
s^{\prime}(x)=\eta^{\prime}(x) q_{1}(x) /\left[\eta(x) q_{1}(x)-q_{2}(x)\right]=2 g^{(\varphi)}(x) G^{(\varphi)}(x) /\left\{1-\left[G^{(\varphi)}(x)\right]^{2}\right\}, \quad x \in \mathbb{R},
$$

and consequently

$$
s(x)=-\log \left\{1-\left[G^{(\varphi)}(x)\right]^{2}\right\}, \quad x \in \mathbb{R} .
$$

Now, according to Theorem .1, $\mathrm{X}$ has density (1.2). 
Corollary 4.2. Let $\mathrm{X}: \Omega \rightarrow \mathbb{R}$ be a continuous random variable and let $\mathrm{q}_{1}(\mathrm{x})$ be as in Proposition 4.1 . The random variable $\mathrm{X}$ has PDF (1.2) if and only if there exist functions $\mathrm{q}_{2}$ and $\eta$ defined in Theorem 1 satisfy the following differential equation

$$
\eta^{\prime}(x) q_{1}(x) /\left[\eta(x) q_{1}(x)-q_{2}(x)\right]=2 g^{(\varphi)}(x) G^{(\varphi)}(x) /\left\{1-\left[G^{(\varphi)}(x)\right]^{2}\right\}, \quad x \in \mathbb{R} .
$$

Corollary 4.3. The general solution of the differential equation in Corollary 4.2 is

$$
\eta(x)=\left\{1-\left[G^{(\varphi)}(x)\right]^{2}\right\}^{-1}\left[-\int 2 g^{(\varphi)}(x) G^{(\varphi)}(x)\left(q_{1}(x)\right)^{-1} q_{2}(x) d x+D\right],
$$

where $\mathrm{D}$ is a constant. We like to point out that one set of functions satisfying the above differential equation is given in Proposition 4.1 with $\mathrm{D}=\frac{1}{2}$. Clearly, there are other triplets $\left(\mathrm{q}_{1}, \mathrm{q}_{2}, \eta\right)$ which satisfy conditions of Theorem .1 .

\section{Parameter estimation}

Here, we will consider the estimation of the unknown parameters $(\lambda, \theta, \varphi)$ of the new $G$ family from complete samples by maximum likelihood method. Let $x_{1}, \ldots, x_{n}$ be a random sample (rs) from the ZTPBrX-G models with a $(q \times 2)$ parameter vector $\Phi=\left(\lambda, \theta, \varphi^{\top}\right)^{\top}$. The log-likelihood function for $\Phi$ is given by

$$
\begin{aligned}
\ell_{n}(\Phi)= & n \log 2+n \log \theta+n \log \lambda-n \log [-\exp (-\lambda)+1]+\sum_{i=1}^{n} \log g^{(\varphi)}\left(x_{i}\right)+\sum_{i=1}^{n} \log G^{(\varphi)}\left(x_{i}\right) \\
& -3 \sum_{i=1}^{n} \log \left[\bar{G}^{(\varphi)}\left(x_{i}\right)\right]-\lambda \sum_{i=1}^{n}\left[1-\exp \left(-s_{i}^{2}\right)\right]^{\theta}-\sum_{i=1}^{n} s_{i}^{2}+(\theta-1) \sum_{i=1}^{n}\left[1-\exp \left(-s_{i}^{2}\right)\right],
\end{aligned}
$$

where $s_{i}=\frac{G^{(\varphi)}\left(x_{i}\right)}{\bar{G}^{(\varphi)}\left(x_{i}\right)}$. The above log-likelihood function can be maximized numerically by using R (optim), SAS (PROC NLMIXED) or Ox program (sub-routine MaxBFGS), among others. For interval estimation of the parameters, the elements of the $q \times q$ observed information matrix $J(\Phi)$ can be evaluated numerically, where

$$
\begin{aligned}
& \mathrm{u}_{\lambda}=\frac{\partial}{\partial \lambda}\left[\ell_{n}(\Phi)\right]=\frac{n}{\lambda}+\frac{n \exp (-\lambda)}{-\exp (-\lambda)+1}-\sum_{i=1}^{n}\left[1-\exp \left(-s_{i}^{2}\right)\right]^{\theta}, \\
& \mathrm{u}_{\theta}=\frac{\partial}{\partial \theta}\left[\ell_{n}(\Phi)\right]=\frac{n}{\theta}+\sum_{i=1}^{n}\left[1-\exp \left(-s_{i}^{2}\right)\right]-\lambda \sum_{i=1}^{n}\left\{\left[1-\exp \left(-s_{i}^{2}\right)\right]^{\theta} \log \left[1-\exp \left(-s_{i}^{2}\right)\right]\right\},
\end{aligned}
$$

and

$$
\begin{aligned}
\mathrm{U}_{\varphi_{\mathrm{k}}}= & \frac{\partial}{\partial \varphi_{\mathrm{k}}}\left[\ell_{\mathrm{n}}(\Phi)\right]=+\sum_{i=1}^{n} \frac{\mathrm{g}^{\prime}}{\mathrm{g}^{(\varphi)}\left(\mathrm{x}_{\mathrm{i}}\right)}+\sum_{i=1}^{n} \frac{\mathrm{G}^{\prime}}{\mathrm{G}^{(\varphi)}\left(x_{i}\right)}-2 \sum_{i=1}^{n} \mathrm{~m}_{i} s_{i} \\
& -3 \sum_{i=1}^{n} \frac{\overline{\mathrm{G}}^{\prime}}{\overline{\mathrm{G}}^{(\varphi)}\left(x_{i}\right)}-2 \lambda \theta \sum_{i=1}^{n} \mathrm{~m}_{i} s_{i} \frac{\left[1-\exp \left(-s_{i}^{2}\right)\right]^{\theta-1}}{\exp \left(s_{i}^{2}\right)}+2(\theta-1) \sum_{i=1}^{n} \frac{m_{i} s_{i}}{\exp \left(s_{i}^{2}\right)}
\end{aligned}
$$

where

$$
g^{\prime}=\frac{\partial}{\partial \varphi_{k}}\left[g^{(\varphi)}\left(x_{i}\right)\right], G^{\prime}=\frac{\partial}{\partial \varphi_{k}}\left[G^{(\varphi)}\left(x_{i}\right)\right], \bar{G}^{\prime}=\frac{\partial}{\partial{ }_{k}^{\prime}}\left[\bar{G}^{(\varphi)}\left(x_{i}\right)\right], \text { and } m_{i}=\frac{\partial s_{i}}{\partial \varphi_{k}} .
$$

\section{Modeling reliability and medical data using the ZTPBrXW model}

In this section, we provide three applications to show empirically the potentiality of the ZTPBrXW model. In order to compare the fits of the ZTPBrXW distribution with other competing distributions, we consider the Cramér-von Mises $\left(W^{*}\right)$ and the Anderson-Darling $\left(A^{*}\right)$ statistics. These two statistics are widely used to determine how closely a specific CDF fits the empirical distribution of a given data set. These statistics are given by 


$$
W^{*}=(1+1 / 2 n)\left[(1 / 12 n)+\sum_{j=1}^{n}\left[z_{i}-(2 j-1) / 2 n\right]^{2}\right]
$$

and

$$
A^{*}=\left\{n+\frac{1}{n} \sum_{j=1}^{n}(2 j-1) \log \left[z_{i}\left(1-z_{n-j+1}\right)\right]\right\}\left(1+\frac{9}{4 n^{2}}+\frac{3}{4 n}\right),
$$

respectively, where $z_{i}=F\left(y_{j}\right)$ and the $y_{j}$ 's values are the ordered observations. The smaller these statistics are, the better the fit. The required computations are carried out using the $\mathrm{R}$ software. The MLEs and the corresponding standard errors (in parentheses) of the model parameters are given in Tables 1, 3, and 5. The numerical values of the statistics $W^{*}$ and $A^{*}$ are listed in Tables 2,4 , and 6 . The histograms of the two data sets and the estimated PDF of the proposed model are displayed in Figures 2 and 3.

\subsection{Application 1}

The first real data set represents the data on failure times of 84 aircraft windshield given in [28]. The data are: $0.040,1.866,2.385,3.443,0.301,1.876,2.481,3.467,0.309,1.899,2.610,3.478,0.557,1.911,2.625$, $4.570,1.652,2.300,3.344,4.602,1.757,3.578,0.943,1.912,2.632,3.595,1.303,2.089,2.902,4.167,1.432$, $4.376,1.615,2.223,3.114,4.449,1.619,2.097,2.934,4.240,1.480,2.135,2.962,4.255,1.505,2.154,2.964,4.278$, $1.506,2.190,3.000,4.305,1.070,1.914,2.646,3.699,1.124,1.981,2.661,3.779,1.248,2.010,2.224,3.117,4.485$, $1.652,2.229,3.166,2.688,3.924,1.281,2.038,2.823,4.035,1.281,2.085,2.890,4.121,1.568,2.194,3.103,2.324$, 3.376, 4.663. Here, we shall compare the fits of the ZTPBrXW distribution with those of other competitive models, namely: Marshall Olkin extended-Weibull (MOEW):

$$
f_{M O E W}^{(\gamma, \beta, \alpha)}(x)=\alpha \beta \gamma^{\beta} x^{\beta-1}\left\{1-(1-\alpha) \exp \left[-(\gamma x)^{\beta}\right]\right\}^{-2} \exp \left[-(\gamma x)^{\beta}\right]
$$

Gamma-Weibull (GaW) ([30])

$$
f_{\mathrm{GaW}}^{(\alpha, \beta, \gamma)}(x)=\beta \alpha^{\gamma / \beta+1} \Gamma^{-1}(1+\gamma / \beta) \chi^{\beta+\gamma-1} \exp \left[-\alpha x^{\beta}\right]
$$

Kumaraswamy-Weibull (KwW) ([11])

$$
f_{\mathrm{KwW}}^{(\alpha, \beta, a, b)}(x)=a b \beta \alpha^{\beta} x^{\beta-1}\left\{1-\exp \left[-(\alpha x)^{\beta}\right]\right\}^{a-1} \exp \left[-(\alpha x)^{\beta}\right]\left\{1-\left\{1-\exp \left[-(\alpha x)^{\beta}\right]\right\}^{a}\right\}^{b-1} ;
$$

Weibull-Fréchet (WFr) ([4])

$$
f_{W F r}^{(\alpha, \beta, a, b)}(x)=a b \beta \alpha^{\beta} x^{\beta-1}\left\{1-\exp \left[-(\alpha x)^{\beta}\right]\right\}^{a-1} \exp \left[-(\alpha x)^{\beta}\right]\left\{1-\left\{1-\exp \left[-(\alpha x)^{\beta}\right]\right\}^{a}\right\}^{b-1} ;
$$

Beta-Weibull (BW) ([24])

$$
f_{B W}^{(\alpha, \beta, a, b)}(x)=\beta \alpha^{\beta} B^{-1}(a, b) x^{\beta-1}\left\{1-\exp \left[-(\alpha x)^{\beta}\right]\right\}^{a-1} \exp \left[-b(\alpha x)^{\beta}\right] ;
$$

Transmuted modified-Weibull (TMW) ([22])

$$
\mathrm{f}_{\mathrm{TMW}}^{(\alpha, \beta, \gamma, \lambda)}(\mathrm{x})=\left(\alpha+\gamma \beta \chi^{\beta-1}\right)\left[1-\lambda+2 \lambda \exp \left(-\alpha x-\gamma \chi^{\beta}\right)\right] \exp \left[-\alpha \chi-\gamma x^{\beta}\right],|\lambda| \leqslant 1 ;
$$

Kumaraswamy transmuted-Weibull (KwTW) ([2])

$$
\begin{aligned}
f_{\mathrm{K} w \mathrm{TW}}^{(\alpha, \beta, \lambda, a, b)}(x)= & \operatorname{ab} \beta \alpha^{\beta} x^{\beta-1}\left(1+\lambda-2 \lambda\left\{1-\exp \left[-(\alpha x)^{\beta}\right]\right\}\right) \exp \left[-(\alpha x)^{\beta}\right] \\
& \times\left[\left\{1-\exp \left[-(\alpha x)^{\beta}\right]\right\}\left(1+\lambda-\lambda\left\{1-\exp \left[-(\alpha x)^{\beta}\right]\right\}\right)\right]^{\mathrm{a}-1}
\end{aligned}
$$




$$
\times\left[1-\left((1+\lambda)\left\{1-\exp \left[-(\alpha x)^{\beta}\right]\right\}-\lambda\left\{1-\exp \left[-(\alpha x)^{\beta}\right]\right\}^{2}\right)^{a}\right]^{b-1}
$$

Modified beta-Weibull (MBW) ([21])

$$
\begin{aligned}
f_{M B W}^{(\alpha, \beta, a, b, c)}(x)= & \beta \gamma^{a} \alpha^{-\beta} B^{-1}(a, b) x^{\beta-1} \exp \left[-b\left(\frac{x}{\alpha}\right)^{\beta}\right]\left\{1-\exp \left[-\left(\frac{x}{\alpha}\right)^{\beta}\right]\right\}^{a-1} \\
& \times\left(1-(1-\gamma)\left\{1-\exp \left[-b\left(\frac{x}{\alpha}\right)^{\beta}\right]\right\}\right)^{-(a+b)}
\end{aligned}
$$

Mcdonald-Weibull (McW) ([10]),

$$
f_{M c W}^{(\alpha, \beta, a, b, c)}(x)=\beta c \alpha^{\beta} B^{-1}(a / c, b) \frac{x^{\beta-1}\left\{1-\exp \left[-(\alpha x)^{\beta}\right]\right\}^{a-1} \exp \left[-(\alpha x)^{\beta}\right]}{\left(1-\left\{1-\exp \left[-(\alpha x)^{\beta}\right]\right\}^{c}\right)^{1-b}} ;
$$

and Transmuted exponentiated generalized Weibull (TExGW) ([37])

$$
f_{\operatorname{TExGW}}^{(\alpha, \beta, \lambda, a, b)}(x)=a b \beta \alpha^{\beta} \frac{x^{\beta-1}\left\{1-\exp \left[-a(\alpha x)^{\beta}\right]\right\}^{b-1} \exp \left[-a(\alpha x)^{\beta}\right]}{\left(1+\lambda-2 \lambda\left\{1-\exp \left[-a(\alpha x)^{\beta}\right]\right\}^{b}\right)^{-1}},|\lambda| \leqslant 1 .
$$

All the above PDFs are for $x>0$.

Based on Table 2 and Figure 3 we conclude that the new lifetime model provides adequate fits as

\begin{tabular}{|c|c|}
\hline Distribution & Estimates \\
\hline $\operatorname{ZTPBrXW}_{(\lambda, \theta, \alpha, \beta)}$ & $\begin{array}{c}-0.0277,1.189885,2.8387796,0.4341143 \\
(1.49708),(0.70545),(1.1007985),(0.095563)\end{array}$ \\
\hline $\operatorname{MOEW}_{(\gamma, \beta, \alpha)}$ & $\begin{array}{l}488.89946,0.283246,1261.966 \\
(189.358),(0.013),(351.073)\end{array}$ \\
\hline $\operatorname{GaW}_{(\alpha, \beta, \gamma)}$ & $\begin{array}{l}2.376973,0.848094,3.534401 \\
(0.378),(0.0005296),(0.665)\end{array}$ \\
\hline $\mathrm{KwW}_{(\alpha, \beta, a, b)}$ & $\begin{array}{l}14.4331,0.2041,34.6599,81.8459 \\
(27.095),(0.042),(17.527),(52.014)\end{array}$ \\
\hline $\operatorname{WFr}_{(\alpha, \beta, a, b)}$ & $\begin{array}{c}630.9384,0.3024,416.0971,1.1664 \\
(697.942),(0.032),(232.359),(0.357)\end{array}$ \\
\hline $\mathrm{BW}_{(\alpha, \beta, a, b)}$ & $\begin{array}{l}1.36,0.2981,34.1802,11.4956 \\
(1.002),(0.06),(14.838),(6.73)\end{array}$ \\
\hline $\operatorname{TMW}_{(\alpha, \beta, \gamma, \lambda)}$ & $\begin{array}{l}0.2722,1,4.6 \times 10^{-6}, 0.4685 \\
(0: 014),\left(5.2 \times 10^{-5}\right),\left(1.9 \times 10^{-4}\right),(0.165)\end{array}$ \\
\hline $\operatorname{KwTW}_{(\alpha, \beta, \lambda, a, b)}$ & $\begin{array}{l}27.7912,0.178,0.4449,29.5253,168.0603 \\
(33.401),(0.017),(0.609),(9.792),(129.165)\end{array}$ \\
\hline $\operatorname{MBW}_{(\alpha, \beta, a, b, c)}$ & $\begin{array}{c}10.1502,0.1632,57.4167,19.3859,2.0043 \\
(18.697),(0.019),(14.063),(10.019),(0.662)\end{array}$ \\
\hline $\operatorname{McW}_{(\alpha, \beta, a, b, c)}$ & $\begin{array}{l}1.9401,0.306,17.686,33.6388,16.7211 \\
(1.011),(0: 045),(6.222),(19.994),(9.722)\end{array}$ \\
\hline $\operatorname{TExGW}_{(\alpha, \beta, \lambda, a, b)}$ & $\begin{array}{l}4.2567,0.1532,0.0978,5.2313,1173.3277 \\
(33.401),(0: 017),(0.609),(9.792)\end{array}$ \\
\hline
\end{tabular}
compared to other Weibull models with small values for $W^{*}$ and $A^{*}$. The proposed lifetime model is much better than the MOEW, GaW, KwW, WFr, BW, TMW, KwTW, MBW, McW, TExGW models, and a good alternative to these models.

Table 1: MLEs (standard errors in parentheses) for data set $\mathbf{I}$. 
Table 2: $W^{*}$ and $A^{*}$ for data set $\mathbf{I}$.

\begin{tabular}{|l|c|c|}
\hline \multicolumn{3}{|c|}{ Table 2: $W^{*}$ and $A^{*}$ for data set I. } \\
\hline $\operatorname{ZTPBrXW}_{(\lambda, \theta, \alpha, \beta)}$ & $\mathbf{0 . 0 6 3 9 6}$ & $\mathbf{0 . 6 2 6 6 8}$ \\
\hline $\operatorname{MOEW}_{(\gamma, \beta, \alpha)}$ & 0.39953 & 4.44766 \\
\hline $\operatorname{GaW}_{(\alpha, \beta, \gamma)}$ & 0.25533 & 1.94887 \\
\hline $\operatorname{KWW}_{(\alpha, \beta, a, b)}$ & 0.18523 & 1.50591 \\
\hline $\operatorname{WFr}_{(\alpha, \beta, a, b)}$ & 0.25372 & 1.95739 \\
\hline $\operatorname{BW}_{(\alpha, \beta, a, b)}$ & 0.46518 & 3.21973 \\
\hline $\operatorname{TMW}_{(\alpha, \beta, \gamma, \lambda)}$ & 0.80649 & 11.20466 \\
\hline $\operatorname{KWTW}_{(\alpha, \beta, \lambda, a, b)}$ & 0.16401 & 1.36324 \\
\hline $\operatorname{MBW}_{(\alpha, \beta, a, b, c)}$ & 0.47172 & 3.26561 \\
\hline $\operatorname{McW}_{(\alpha, \beta, a, b, c)}$ & 0.1986 & 1.59064 \\
\hline $\operatorname{TExGW}_{(\alpha, \beta, \lambda, a, b)}$ & 1.00791 & 6.23321 \\
\hline
\end{tabular}
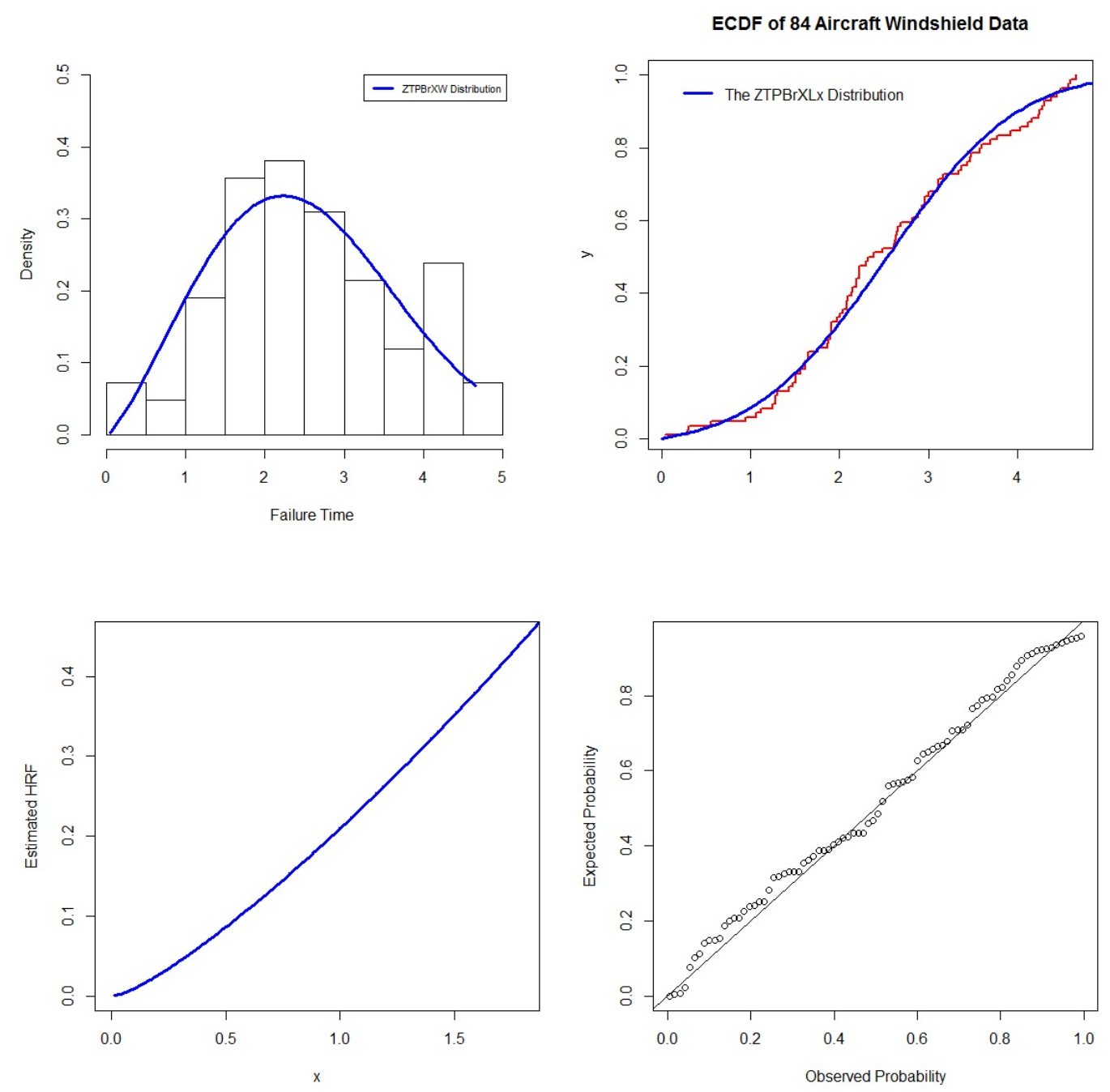

Figure 3: Estimated PDF, CDF, HRF, and PP plot for data set I. 


\subsection{Application 2}

This data set represents the remission times (in months) of a random sample of 128 bladder cancer patients. This data is given by: $0.08,2.09,3.48,4.87,6.94,8.66,13.11,23.63,0.20,2.23,3.52,4.98,6.97$, $9.02,13.29,0.40,2.26,3.57,5.06,7.09,9.22,13.80,25.74,0.50,2.46,3.64,5.09,7.26,9.47,14.24,25.82,0.51$, $2.54,3.70,5.17,5.41,7.62,10.75,16.62,43.01,1.19,2.75,4.26,5.41,7.63,17.12,46.12,1.26,2.83,4.33,5.49$, 7.66, 11.25, 17.14, 79.05, 1.35, 2.87, 5.62, 7.87, 11.64, 17.36, 1.40, 3.02, 4.34, 5.71, 7.28, 9.74, 14.76, 26.31, 0.81, $2.62,3.82,5.32,7.32,10.06,14.77,32.15,2.64,3.88,5.32,7.39,10.34,14.83,34.26,0.90,2.69,4.18,5.34,7.59$, $10.66,15.96,36.66,1.05,2.69,4.23,7.93,11.79,18.10,1.46,4.40,5.85,8.26,11.98,19.13,1.76,3.25,4.50,6.25$, $8.37,12.02,2.02,3.31,4.51,6.54,8.53,12.03,20.28,2.02,3.36,6.76,12.07,21.73,2.07,3.36,6.93,8.65,12.63$, 22.69. We compare the fits of the ZTPBrXW distribution with other competitive models, namely the TMW, MBW, Transmuted additive Weibull distribution (TAW):

$$
f_{\text {TAW }}^{(\alpha, \beta, \gamma, \theta, \lambda)}(x)=\left(\alpha \theta x^{\theta-1}+\gamma \beta x^{\beta-1}\right) e^{-\left(\alpha x^{\theta}+\gamma x^{\beta}\right)}\left\{1-\lambda+2 \lambda e^{-\left(\alpha x^{\theta}+\gamma x^{\beta}\right)}\right\} ;
$$

the Exponentiated transmuted generalized Rayleigh (ETGR) ([3])

$$
f_{\mathrm{ETGR}}^{(\alpha, \beta, \delta, \lambda)}(x)=2 \alpha \delta \beta^{2} \frac{x e^{-(\beta x)^{2}}\left\{1+\lambda-\lambda\left(1-e^{-(\beta x)^{2}}\right)^{\alpha}\right\}^{\delta-1}}{\left[1-e^{-(\beta x)^{2}}\right]^{-(\alpha \delta-1)}\left[1+\lambda-2 \lambda\left(1-e^{-(\beta x)^{2}}\right)^{\alpha}\right]^{-1}},|\lambda| \leqslant 1 .
$$

Above PDFs are for $x>0$.
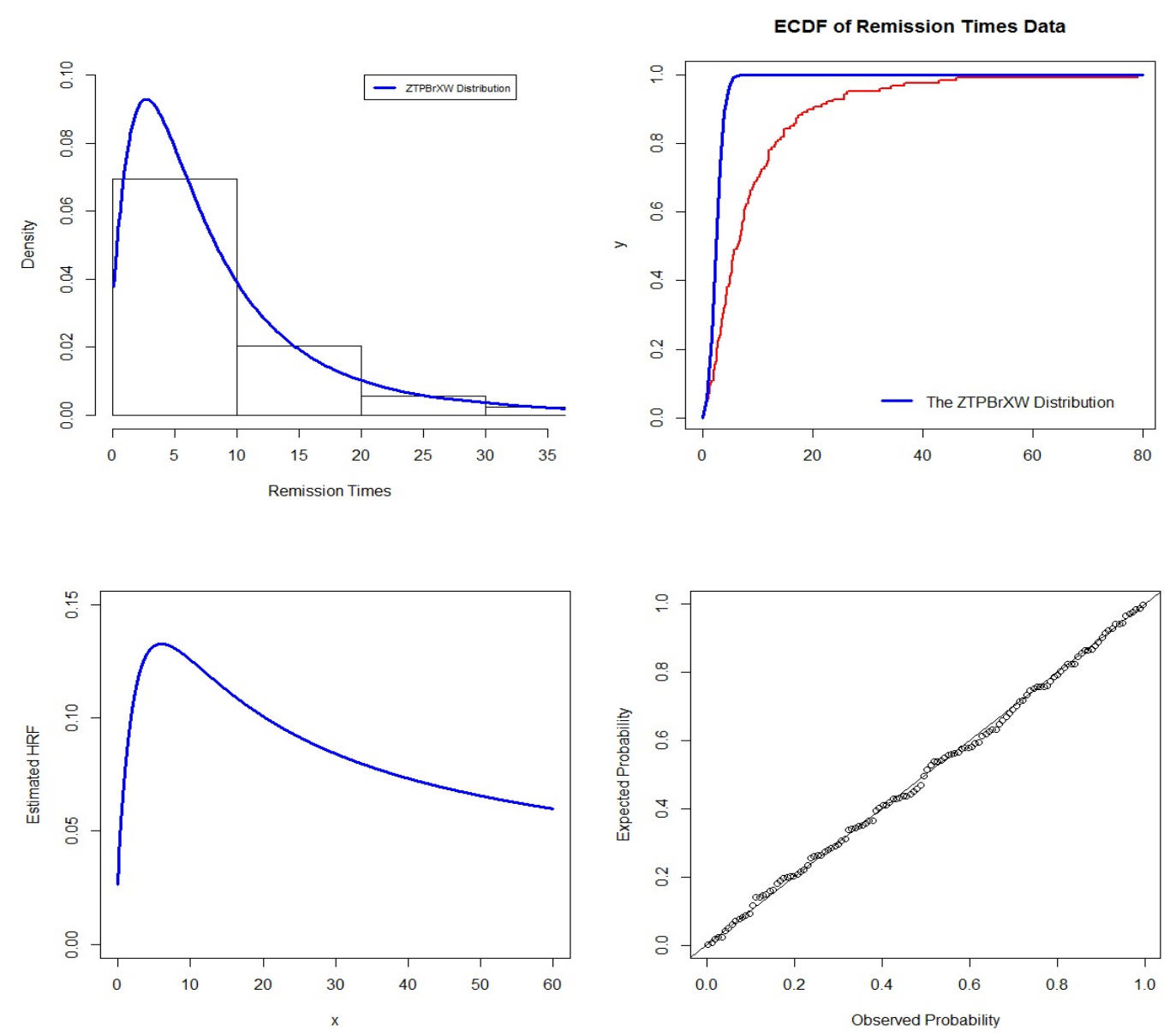

Figure 4: Estimated PDF, CDF, HRF and PP plot for data set II. 
Table 3: MLEs (standard errors in parentheses) for data set II.

\begin{tabular}{|l|l|}
\hline Distribution & Estimates \\
\hline ZTPBrXW $_{(\lambda, \theta, \alpha, \beta)}$ & $-4.3130,4.8119,1.2414,0.10667$ \\
& $(2.4512),(5.02761),(0.1837),(0.0188)$ \\
\hline $\mathrm{W}_{(\alpha, \beta)}$ & $9.5593,1.0477$ \\
& $(0.853),(0.068)$ \\
\hline TMW $_{(\alpha, \beta, \gamma, \lambda)}$ & $0.1208,0.8955,0.0002,0.2513$ \\
& $(0.024),(0.626),(0.011),(0.407)$ \\
\hline $\operatorname{MBW}_{(\alpha, \beta, a, b, c)}$ & $0.1502,0.1632,57.4167,19.3859,2.0043$ \\
& $(22.437),(0.044),(37.317),(13.49),(0.789)$ \\
\hline $\operatorname{TAW}_{(\alpha, \beta, \gamma, \theta, \lambda)}$ & $0.1139,0.9722,3.0936 \times 10^{-5}, 1.0065,-0.163$ \\
& $(0.032),(0.125),\left(6.106 \times 10^{-3}\right),(0.035),(0.28)$ \\
\hline ETGR $_{(\alpha, \beta, \delta, \lambda)}$ & $7.3762,0.0473,0.0494,0.118$ \\
& $(5.389),\left(3.965 \times 10^{-3}\right),(0.036),(0.26)$ \\
\hline
\end{tabular}

Table 4: $W^{*}$ and $A^{*}$ for data set II.

\begin{tabular}{|l|c|c|}
\hline Distribution & $W^{*}$ & $A^{*}$ \\
\hline $\operatorname{ZTPBrXW}_{(\lambda, \theta, \alpha, \beta)}$ & $\mathbf{0 . 0 1 9 2 9 8}$ & $\mathbf{0 . 1 2 4 0 8 1}$ \\
\hline $\mathrm{W}_{(\alpha, \beta)}$ & 0.10553 & 0.66279 \\
\hline $\operatorname{TMW}_{(\alpha, \beta, \gamma, \lambda)}$ & 0.12511 & 0.76028 \\
\hline $\operatorname{MBW}_{(\alpha, \beta, a, b, c)}$ & 0.10679 & 0.72074 \\
\hline $\operatorname{TAW}_{(\alpha, \beta, \gamma, \theta, \lambda)}$ & 0.11288 & 0.70326 \\
\hline $\operatorname{ETGR}_{(\alpha, \beta, \delta, \lambda)}$ & 0.39794 & 2.36077 \\
\hline
\end{tabular}

Based on Table 4 and Figure 4 we conclude that the proposed lifetime model is much better than the W, TMW, MBW, TAW, ETGR models with small values for $W^{*}$ and $A^{*}$ in modeling cancer patients data.

\subsection{Application 3}

The real data set corresponds to the survival times (in days) of 72 guinea pigs infected with virulent tubercle bacilli. The data are: 10, 33, 44, 56, 59, 72,74, 77, 92, 93, 96, 100, 100, 102, 105, 107, 107, 108, 108, $108,109,112,113,115,116,120,121,122,122,124,130,134,136,139,144,146,153,159,160,163,163$, $168,171,172,176,183,195,196,197,202,213,215,216,222,230,231,240,245,251,253,254,255,278,293$, $327,342,347,361,402,432,458,555$. We shall compare the fits of the ZTPBrXW distribution with those of other competitive models, namely: Burr X Exponentiated Weibull (BrXEW)

$$
\begin{aligned}
\mathrm{f}_{\operatorname{BrXEW}}^{(\theta, \alpha, \beta)}(x)= & 2 \theta \alpha \beta x^{\beta-1}\left[1-\exp \left(-x^{\beta}\right)\right]^{2 \alpha-1} \exp \left(-x^{\beta}\right) \\
& \times\left[1-\exp \left(-\left\{\frac{\left[1-\exp \left(-x^{\beta}\right)\right]^{\alpha}}{1-\left[1-\exp \left(-x^{\beta}\right)\right]^{\alpha}}\right\}\right)\right]^{\theta-1} \\
& \times\left\{1-\left[1-\exp \left(-x^{\beta}\right)\right]^{\alpha}\right\}^{-3} \exp \left(-\left\{\frac{\left[1-\exp \left(-x^{\beta}\right)\right]^{\alpha}}{1-\left[1-\exp \left(-x^{\beta}\right)\right]^{\alpha}}\right\}^{2}\right)
\end{aligned}
$$

the Weibull-Weibull (WW):

$$
f_{W W}^{(\beta, \gamma, \lambda)}(x)=\exp \left(-\alpha\left\{-\log \left[1-\exp \left(-\lambda x^{\gamma}\right)\right]\right\}^{\beta}\right) ;
$$

the Gamma Exponentiated Exponential (GaEE) ([33])

$$
f_{\mathrm{GaEE}}^{(\lambda, \alpha, \theta)}(x)=\frac{\alpha \theta}{\Gamma(\lambda)} \exp (-\theta x)[1-\exp (-\theta x)]^{\alpha-1}\{-\alpha \log [1-\exp (-\theta x)]\}^{\lambda-1} ;
$$


and Exponential Exponential geometric (EEGc) ([32]) distributions

$$
f_{\mathrm{EEGc}}^{(\alpha, \theta, p)}(x)=\alpha \theta(1-p) \exp (-\theta x)[1-\exp (-\theta x)]^{1-\alpha}\left\{1-p+p[1-\exp (-\theta x)]^{\alpha}\right\}^{-2},
$$

where PDFs are for $x>0$.
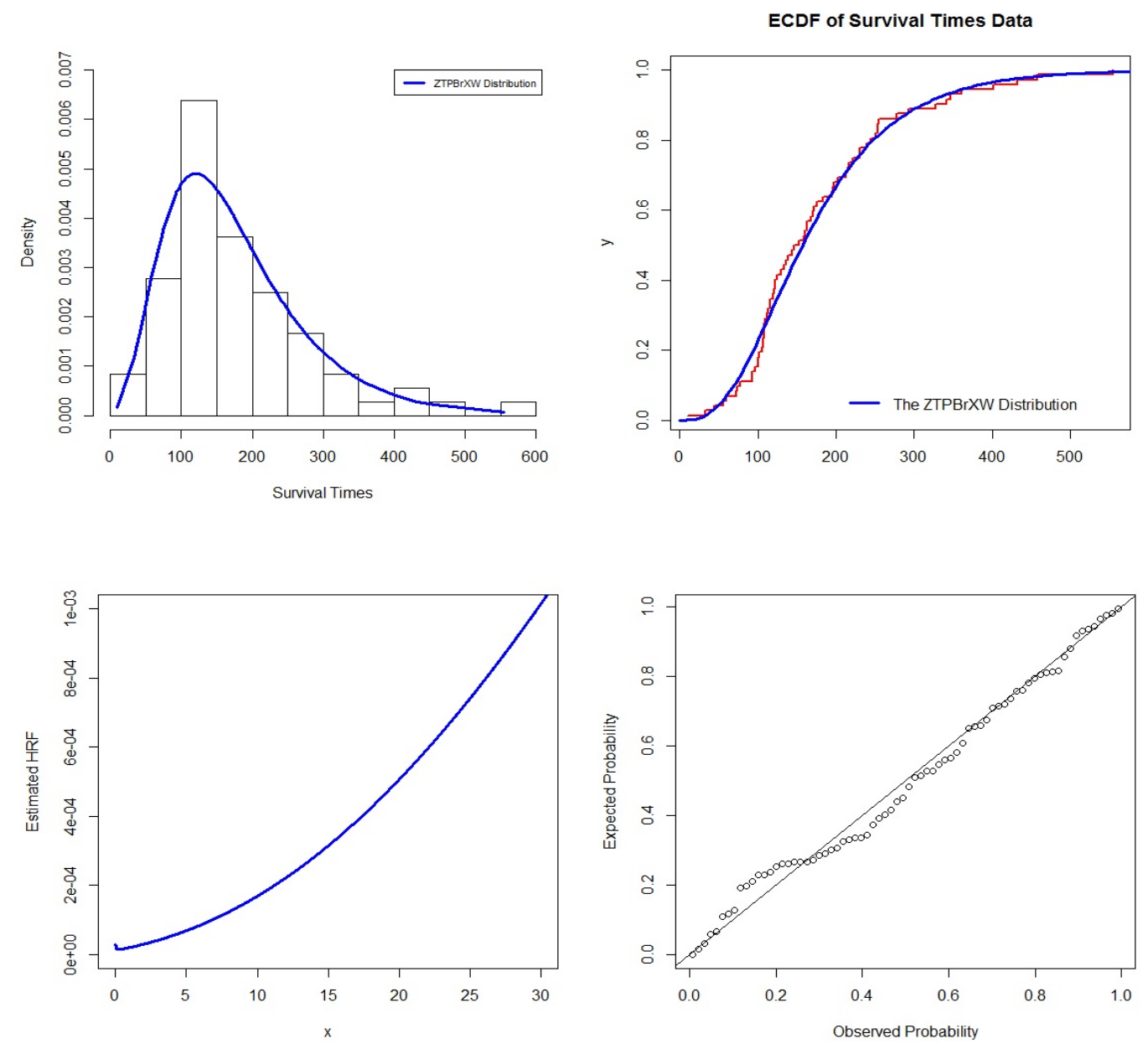

Figure 5: Estimated PDF, CDF, HRF, and PP plot for data set III.

Table 5: MLEs (standard errors in parentheses) for data set III.

\begin{tabular}{|l|l|}
\hline Distribution & Estimates \\
\hline \hline ZTPBrXW $_{(\lambda, \theta, \alpha, \beta)}$ & $-13.8394,0.4565,4.0646,0.14999$ \\
& $(0.000),(0.000),(1.3448),(0.0206)$ \\
\hline $\operatorname{BrXEW}_{(\theta, \alpha, \beta)}$ & $3.18,5.539,0.166$ \\
& $(2.1174),(2.437),(0.0241)$ \\
\hline$W_{(\beta, \gamma, \lambda)}$ & $2.6594,0.6933,0.0270$ \\
& $(0.7129),(0.1707),(0.0193)$ \\
\hline $\operatorname{OWW}_{(\beta, \gamma, \lambda)}$ & $11.1576,0.0881,0.4574$ \\
& $(4.5449),(0.0355),(0.0770)$ \\
\hline $\operatorname{GaEE}_{(\lambda, \alpha, \theta)}$ & $2.1138,2.6006,0.0083$ \\
& $(1.3288),(0.5597),(0.0048)$ \\
\hline $\operatorname{EEGc}_{(\alpha, \theta, p)}$ & $2.5890,0.0004,0.9999$ \\
& $(0.4820), 0.0041),(0.1036)$ \\
\hline
\end{tabular}


Table 6: $W^{*}$ and $A^{*}$ for data set III.

\begin{tabular}{|l|c|c|}
\hline Distribution & $W^{*}$ & $A^{*}$ \\
\hline ZTPBrX-W $_{(\lambda, \theta, \alpha, \beta)}$ & $\mathbf{0 . 0 7 6 9}$ & $\mathbf{0 . 4 7 3 7}$ \\
\hline BrX-EW $_{(\theta, \alpha, \beta)}$ & 0.0907 & 0.5668 \\
\hline W-W \\
$(\beta, \gamma, \lambda)$ & 0.1427 & 0.7811 \\
\hline OW-W $_{(\beta, \gamma, \lambda)}$ & 0.4494 & 2.4764 \\
\hline GaE-E $_{(\lambda, \alpha, \theta)}$ & 0.3150 & 1.7208 \\
\hline EE-Gc $_{(\alpha, \theta, p)}$ & 0.1047 & 0.5789 \\
\hline
\end{tabular}

Based on Table 6 and Figure 5 we conclude that the proposed lifetime model is much better than the BrXEW, WW, OWW, WLogW,GaEE, EEGc models with small values for $W^{*}$ and $A^{*}$ in modeling cancer patients data.

\section{Modeling failure and service times using the ZTPBrXLx model}

In this section, we provide two applications to two real data sets to prove the importance and flexibility of the ZTPBrXLx distribution. We compare the fit of the ZTPBrXLx with competitive models namely: the Burr X Lomax (BrXLx) ([1, 38]):

$$
\mathrm{F}_{\mathrm{BrXLx}}^{(\theta, \lambda, \beta)}(\chi)=\left(1-\exp \left\{-\left[\frac{1-\left(1+x \beta^{-1}\right)^{-\lambda}}{\left(1+x \beta^{-1}\right)^{-\lambda}}\right]^{2}\right\}\right)^{\theta}
$$

ELx model ([17])

$$
\mathrm{F}_{\mathrm{ELx}}^{(\alpha, \beta, \lambda)}(x)=\left[1-\left(1+x \beta^{-1}\right)^{-\lambda}\right]^{\alpha}
$$

the Gamma Lomax (GLx) model ([12]):

$$
\mathrm{F}_{\mathrm{GLx}}^{(\alpha, \beta, \lambda)}(x)=\Gamma^{-1}(\alpha) \Gamma\left(\alpha ; \lambda \log \left[1+x \beta^{-1}\right]\right) ;
$$

the Beta Lomax (BLX) model ([25]):

$$
\mathrm{F}_{\mathrm{BLx}}^{(\alpha, \beta, \theta, \lambda)}(x)=\frac{1}{\mathrm{~B}(\alpha, \theta)} \mathrm{B}\left(1-\left(1+x \beta^{-1}\right)^{-\lambda} ; \alpha, \theta\right) ;
$$

and Lx model ([26]) (for $x>0$ and $\alpha, \beta, \lambda, a>0$ ), where $\Gamma(\cdot)$ is the Gamma function, $\Gamma(\cdot ; \cdot)$ is the incomplete Gamma function, $\mathrm{B}(\cdot, \cdot)$ is the complete beta function and $\mathrm{B}(\cdot ; \cdot, \cdot)$ is the incomplete beta function.

\subsection{Application 4}

This data set represents the data on failure times of 84 aircraft windshield given in Application 1 . From Table 8 and Figure 6, the ZTPBrXLx lifetime model is much better than Gamma Lomax, beta Lomax, exponentiated Lomax and Lomax models so the new model is a good alternative to these models in modeling aircraft windshield data.

\subsection{Application 5}

This real data set represents the data on service times of 63 aircraft windshield given in [28]. The data are: $0.046,1.436,2.592,0.140,1.492,2.600,0.150,1.580,2.670,0.248,1.7190,2.717,0.2800,1.794,2.819$, $0.3130,1.915,2.820,0.389,1.9200,2.878,0.487,1.9630,2.950,0.622,1.978,3.0030,0.9000,2.053,3.1020,0.952$, $2.065,3.3040,0.9960,2.117,3.483,1.0030,2.137,3.500,1.0100,2.141,3.6220,1.085,2.163,3.6650,1.092,2.183$, $3.695,1.1520,2.2400,4.015,1.183,2.3410,4.628,1.2440,2.435,4.806,1.249,2.4640,4.881,1.262,2.5430,5.140$. These data sets were recently studied by Tahir [34] and Abouelmagd [1]. The unit for measurement is 1000 hours for both data sets. From Table 10 and Figure 7, the ZTPBrXLx lifetime model is much better than Gamma Lomax, beta Lomax, exponentiated Lomax and Lomax models so new Lomax model is a good alternative to these models in modeling the service times data. 
Table 7: MLEs (standard errors in parentheses) for data set IV.

\begin{tabular}{|l|l|}
\hline Model & Estimates \\
\hline ZTPBrXLx $(\lambda, \theta, \alpha, \beta)$ & $-5.2837,0.2860,1.553,4.6136$ \\
& $(93.9891),(0.3119),(1.3949),(5.8431)$ \\
\hline $\operatorname{BrXLx}_{(\beta, \theta, \lambda)}$ & $5.237 \times \mathrm{e}^{5}, 8.2291 \times \mathrm{e}^{-1}, 1.1592 \times \mathrm{e}^{5}$ \\
& $(0.000),(0.10511),(5446.8)$ \\
\hline $\operatorname{BLx}_{(\alpha, \beta, \theta, \lambda)}$ & $3.6036,118.8374,33.6387,4.8307$ \\
& $(0.6187),(63.7145),(9.2382),(429.000)$ \\
\hline $\operatorname{ELx}_{(\alpha, \beta, \lambda)}$ & $3.6261,26257.681,20074.51$ \\
& $(0.6236),(99.7417),(2041.826)$ \\
\hline $\operatorname{GLx}_{(\alpha, \beta, \lambda)}$ & $3.5876,37029,52001$ \\
& $(0.5133),(81.1644),(7955)$ \\
\hline $\operatorname{Lx}_{(\beta, \lambda)}$ & 131789,51425 \\
& $(296.120),(5933.49)$ \\
\hline
\end{tabular}

Table 8: $W^{*}$ and $A^{*}$ for data set IV.

\begin{tabular}{|l|c|c|}
\hline Model & $W^{*}$ & $A^{*}$ \\
\hline $\operatorname{ZTPBrXLx}_{(\lambda, \theta, \alpha, \beta)}$ & $\mathbf{0 . 0 7 4 5 9}$ & $\mathbf{0 . 1 5 3 1}$ \\
\hline $\operatorname{BrXLx}_{(\beta, \theta, \lambda)}$ & 0.0764 & 0.5844 \\
\hline $\operatorname{BLx}_{(\alpha, \beta, \theta, \lambda)}$ & 1.4084 & 0.1680 \\
\hline $\operatorname{ELx}_{(\alpha, \beta, \lambda)}$ & 1.7435 & 0.2194 \\
\hline $\operatorname{GLx}_{(\alpha, \beta, \lambda)}$ & 1.3667 & 0.1619 \\
\hline $\operatorname{Lx}_{(\beta, \lambda)}$ & 1.3976 & 0.1665 \\
\hline
\end{tabular}
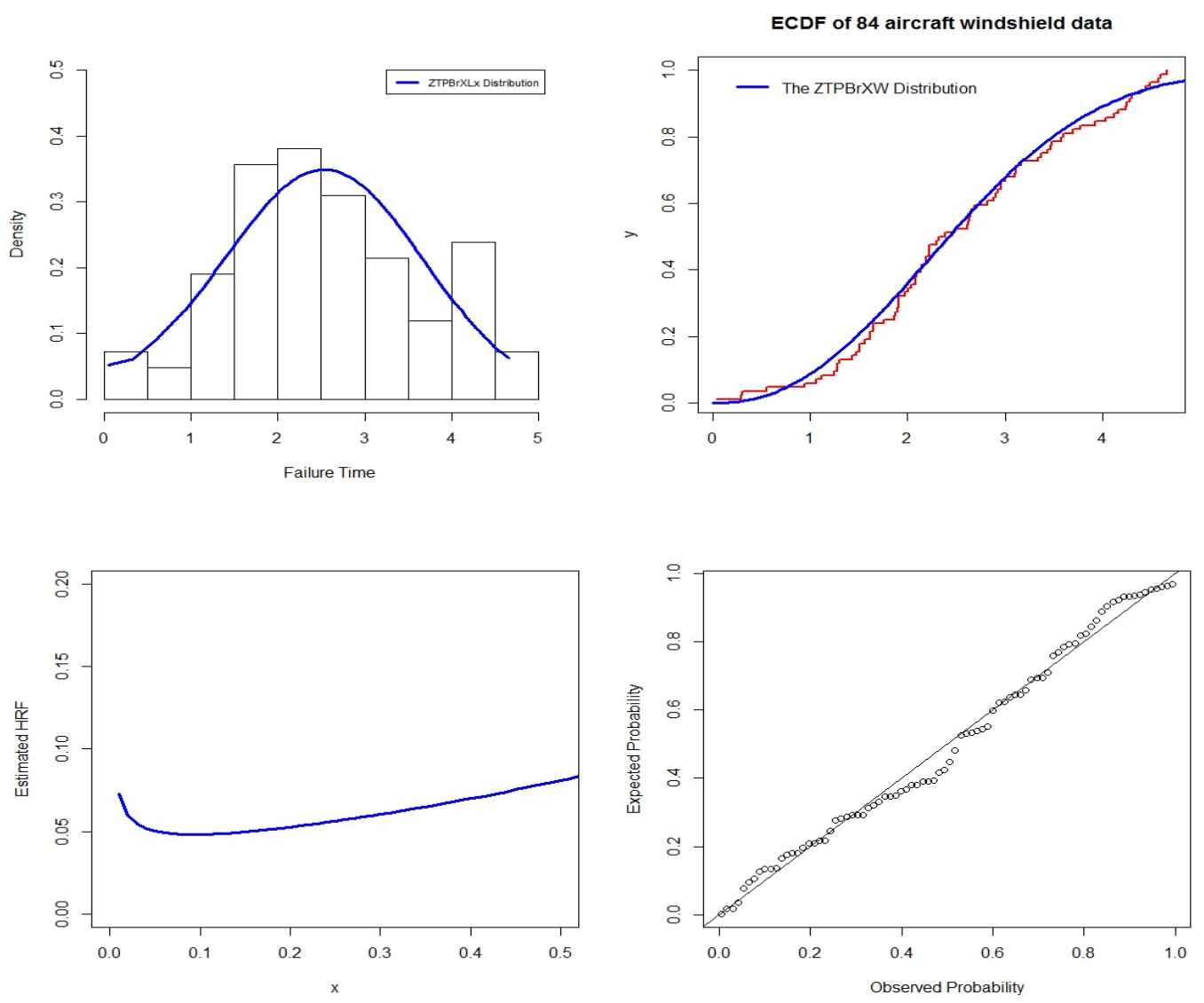

Figure 6: Estimated PDF, CDF, HRF, and PP plot for data set IV. 
Table 9: MLEs (standard errors in parentheses) for data set V.

\begin{tabular}{|l|l|}
\hline Model & Estimates \\
\hline ZTPBrXLx$_{(\lambda, \theta, \alpha, \beta)}$ & $-1.4557,0.4652,1.3517,3.9449$ \\
& $(2.3026),(0.2150), 91.4998),(6.4402)$ \\
\hline $\operatorname{BrXLx}_{(\beta, \theta, \lambda)}$ & $0.6467,0.5987,1.6211$ \\
& $(0.0475),(0.390),(0.959)$ \\
\hline $\operatorname{BLx}_{(\alpha, \beta, \theta, \lambda)}$ & $1.9218,169.5800,31.2595,4.9685$ \\
& $(0.3185),(339.2068),(316.8413),(50.5279)$ \\
\hline $\operatorname{ELx}_{(\alpha, \beta, \lambda)}$ & $1.9145,32881.9,22971.2$ \\
& $(0.3483),(162.2230),(3209.5)$ \\
\hline $\operatorname{GLx}_{(\alpha, \beta, \lambda)}$ & $1.9073,39197.6,35842.4$ \\
& $(0.3214),(151.6530),(6945)$ \\
\hline $\operatorname{Lx}_{(\beta, \lambda)}$ & 207019,99269 \\
& $(301.2370),(11863.5222)$ \\
\hline
\end{tabular}

Table 10: $W^{*}$ and $A^{*}$ for data set $\mathbf{V}$.

\begin{tabular}{|l|c|c|}
\hline Model & $W^{*}$ & $A^{*}$ \\
\hline $\operatorname{ZTPBrXLx}_{(\lambda, \theta, \alpha, \beta)}$ & $\mathbf{0 . 0 3 3 7}$ & $\mathbf{0 . 1 2 2 9 5}$ \\
\hline $\operatorname{BrXLx}_{(\beta, \theta, \lambda)}$ & 0.0876 & 0.5278 \\
\hline $\operatorname{BLx}_{(\alpha, \beta, \theta, \lambda)}$ & 1.1336 & 0.1872 \\
\hline $\operatorname{ELx}_{(\alpha, \beta, \lambda)}$ & 1.2331 & 0.2037 \\
\hline $\operatorname{GLx}_{(\alpha, \beta, \lambda)}$ & 1.1121 & 0.2038 \\
\hline $\operatorname{Lx}_{(\beta, \lambda)}$ & 1.1265 & 0.1861 \\
\hline
\end{tabular}
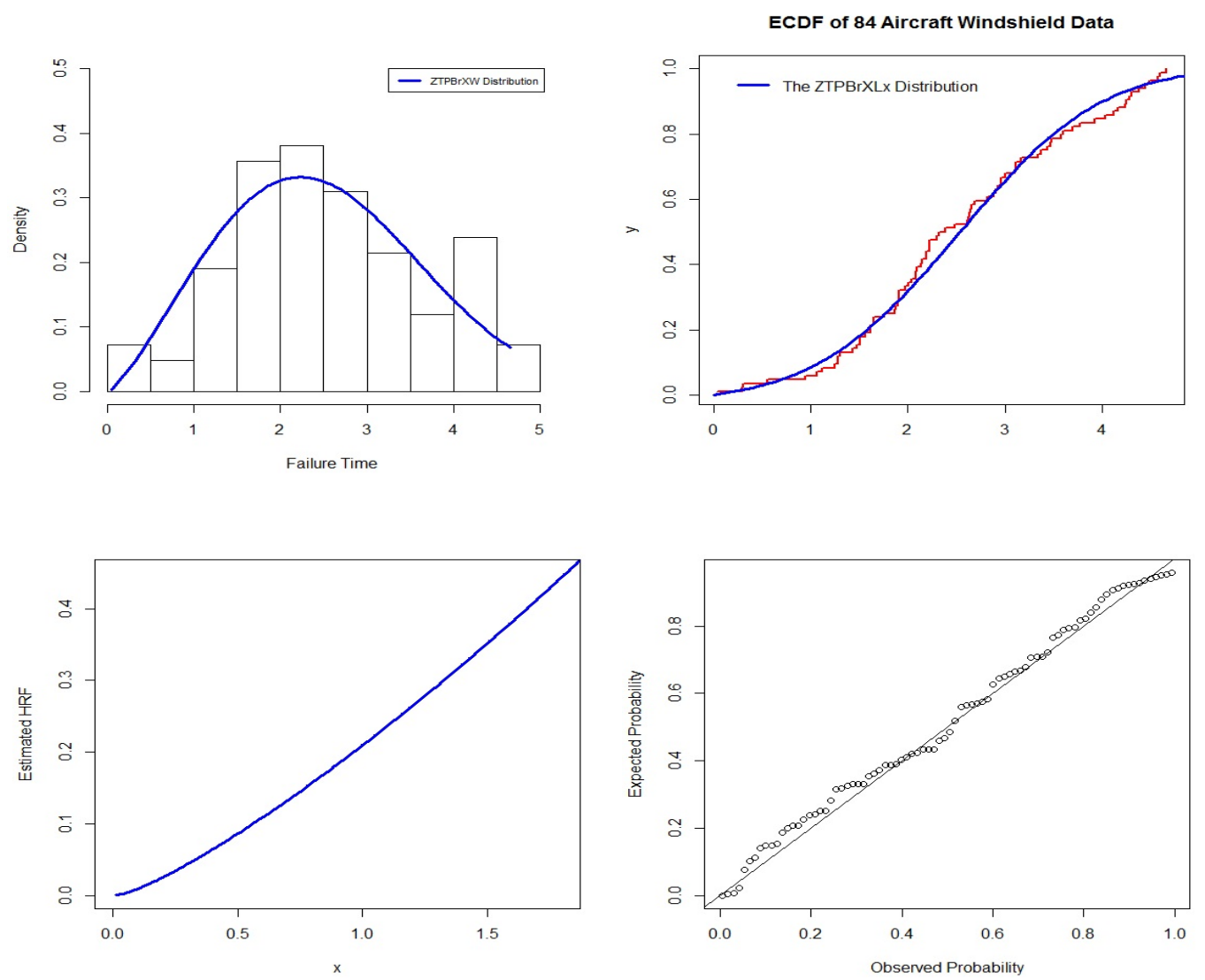

Figure 7: Estimated PDF, CDF, HRF and PP plot for data set V. 


\section{A modified goodness-of- fit test for ZTPBrX-G family in complete data case}

Checking if a series of observations can be modeled by a probability distribution is one of the most important problems in statistical analysis. To validate the choice of a model, we use what we call tests of goodness-of-fit. Researchers have been studying this problem since the beginning of the 20th century when Karl Pearson proposed the famous chi-square goodness-of-fit test (GOF) test. We propose, as a first step, the construction of Nikulin-Rao-Robson (NRR) based chi-squared fit tests for the ZTPBrX-G family in the case of complete data. This test is based on the NRR statistic separately proposed by Nikulin [29] and Rao and Robson [31]. As a second step, an application to real data study has been proposed to show the maneuverability of the proposed test. For more details about non-parametric tests and chi-squared type tests see $[6,7]$.

\subsection{Nikulin-Rao-Robson statistic test for complete data}

The well-known Nikulin-Rao-Robson (NRR) statistic $\left(Y_{n}^{2}\left(\widehat{\Theta}_{n}\right)\right)$ is one of famous modified classical chisquared goodness-of-fit test (GOF), that was introduced by Nikulin [29] and Rao-Robson [31] ([14, 35, 36]). It uses the maximum likelihood estimation on the initial data. Suppose that $X=\left(X_{1}, X_{2}, \ldots, X_{n}\right)^{\top}$ is a simple sample of $n$ independent and identically distributed random variables.

The problem is to test the null composite hypothesis $H_{0}$ : that the i.i.d. random variables $X_{1}, X_{2}, \ldots, X_{n}$ follow the same distribution, i.e.,

$$
P\left(X_{i} \leqslant x\right)=F(x, \Theta), x \in \mathbb{R}, \Theta=\left(\theta_{1}, \theta_{2}, \ldots, \theta_{s}\right)^{\top} .
$$

Nikulin [29] and Rao and Robson [31] proposed the NRR $Y_{n}^{2}$ statistic defined as follows. Let

$$
-\infty=a_{0}<a_{1}<\cdots<a_{r-1}<a_{r}=+\infty
$$

be the boundary points of the grouping intervals $I_{1}, I_{2}, \ldots, I_{r}$ in $(-\infty,+\infty)$. The vector of probabilities is $p(\Theta)=\left(p_{1}(\Theta), p_{2}(\Theta), \ldots, p_{r}(\Theta)\right)^{\top}$, with

$$
p_{j}(\Theta)=\int_{a_{j-1}}^{a_{j}} f(x, \Theta) d x, j=1,2, \ldots, r .
$$

So, the boundaries of intervals $a_{j}$ are given by

$$
a_{j}=F^{-1}\left(\frac{j}{r}\right), j=1, \ldots, r-1 .
$$

If $v_{j}=\left(v_{1}, v_{2}, \ldots, v_{r}\right)^{\top}$ is the vector of frequencies obtained by the grouping of data in the intervals $I_{j}$, where

$$
v_{j}=\sum_{i=1}^{n} I_{\left\{x_{i} \in I_{j}\right\}} j=1, \ldots, r,
$$

which lies in each of the successive intervals

$$
\left[a_{0}, a_{1}\right), \ldots,\left[a_{r-1}, a_{r}\right) .
$$

The NRR statistic with $\widehat{\Theta}_{n}$ as a maximum likelihood estimator of the parameter vector $\Theta$, is given by

$$
Y_{n}^{2}\left(\widehat{\Theta}_{n}\right)=X_{n}^{2}\left(\widehat{\Theta}_{n}\right)+\frac{1}{n} L^{\top}\left(\widehat{\Theta}_{n}\right)\left(I\left(\widehat{\Theta}_{n}\right)-J\left(\widehat{\Theta}_{n}\right)\right)^{-1} L\left(\widehat{\Theta}_{n}\right) .
$$

The Pearson's statistic is $X_{n}^{2}(\Theta)=X_{n}^{\top}(\Theta) X_{n}(\Theta)$, where

$$
X_{n}(\Theta)=\left(\frac{v_{1}-n p_{1}(\Theta)}{\sqrt{n p_{1}(\Theta)}}, \frac{v_{2}-n p_{2}(\Theta)}{\sqrt{n p_{2}(\Theta)}}, \cdots, \frac{v_{r}-n p_{r}(\Theta)}{\sqrt{n p_{r}(\Theta)}}\right)^{\top} .
$$


The $\mathrm{I}\left(\Theta_{n}\right)$ represents the Fisher information matrix, and

$$
l(\Theta)=\left(l_{1}(\Theta), \ldots, l_{s}(\Theta)\right)^{\top} ; \text { with } l_{k}(\Theta)=\sum_{i=1}^{r} \frac{v_{i}}{p_{i}} \frac{\partial p_{i}(\Theta)}{\partial \Theta_{k}} .
$$

$J(\Theta)$ is the information matrix for the grouped data defined by $J(\Theta)=B(\Theta)^{T} B(\Theta)$, where

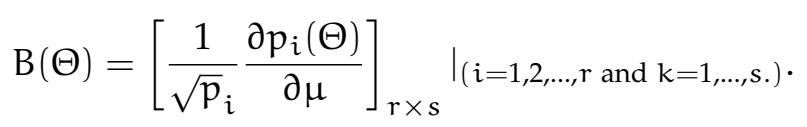

Under the null hypothesis $\mathrm{H}_{0}$; the NRR $\left(\mathrm{Y}_{n}^{2}\left(\widehat{\Theta}_{n}\right)\right)$ statistic follows a chi-square distribution with $r-1$ degrees of freedom. So, for any fixed $x>0$, we have:

$$
\lim _{n \rightarrow \infty} \mathbf{P}\left(Y_{n}^{2}\left(\widehat{\Theta}_{n}\right) \geqslant x\right)=\mathbf{P}\left(\chi_{r-1}^{2} \geqslant x\right) .
$$

\subsection{Goodness of fit test for ZTPBrXW and ZTPBrXLx models}

Let us consider the null hypothesis $\mathrm{H}_{0}$ according to which the distribution of the sample $\mathbb{X}=$ $\left(X_{1}, X_{2}, \ldots, X_{n}\right)^{\top}$ is given by

$$
\begin{aligned}
H_{0}:\left.P\left(X_{i} \leqslant \chi\right)\right|_{\left[\Theta=(\alpha, \lambda, \beta, \theta)^{\top}, \chi \geqslant 0\right]} & =F_{Z \operatorname{TPBrXW}}(\chi, \Theta) \\
& =\frac{1-\exp \left(-\lambda\left\{1-\exp \left[-\left(\left\{\exp \left[(\alpha x)^{\beta}\right]\right\}-1\right)^{2}\right]\right\}^{\theta}\right)}{-\exp (-\lambda)+1},
\end{aligned}
$$

and

$$
\begin{aligned}
H_{0}:\left.P\left(X_{i} \leqslant x\right)\right|_{\left[\Theta=(\alpha, \lambda, \beta, \theta)^{\top}, x \geqslant 0\right]} & =F_{Z T P B r X L x}(x, \Theta) \\
& =\frac{1-\exp \left(-\lambda\left\{1-\exp \left(-\left\{\left[1+\left(x \beta^{-1}\right)\right]^{\alpha}-1\right\}^{2}\right)\right\}^{\Theta}\right)}{-\exp (-\lambda)+1},
\end{aligned}
$$

where $F_{Z T P B r X W}(x, \Theta)$ and $F_{Z T P B r X L x}(x, \Theta)$ are the distribution functions of the ZTPBrX-Weibull and ZTPBrX-Lomax distributions. To support the results obtained in this work and showing the usefulness of the modified chi-square NRR $\left(Y_{\mathfrak{n}}^{2}\left(\widehat{\Theta}_{\mathfrak{n}}\right)\right)$ statistic tests and the applicability of the $\mathrm{F}_{Z T P B r X W}(x, \Theta)$ and $\mathrm{F}_{\mathrm{ZTPBrXLx}}(x, \Theta)$ models, we apply it to two real data; which represents the data of failure times of 84 aircraft windshield given in [28] and the remission times (in months) of a random sample of 128 bladder cancer patients.

\subsubsection{Example 1}

To test the null hypothesis $\mathrm{H}_{0}$ that the aircraft windshield ([28]) data arise from a Zero Truncated Poisson Burr X Weibull distribution, we use the NRR statistic explained above. Using the R software and the Barzilai-Borwein (BB) algorithm, we first calculate for $r=9$ intervals, the maximum likelihood estimates.

$$
\widehat{\lambda}=0.2318, \widehat{\theta}=0.2239, \widehat{\alpha}=2.0076, \widehat{\beta}=0.1106 \text {. }
$$

Then, the values of the NRR statistic tests is : $Y_{n}^{2}\left(\widehat{\Theta}_{n}\right)=16.9834$. We see that

$$
Y_{n}^{2}\left(\widehat{\Theta}_{n}\right) \leqslant \chi_{r-1, \alpha=0.05}^{2}=15.50731 \text {. }
$$

On the other hand we have

$$
Y_{n}^{2}\left(\widehat{\Theta}_{n}\right) \leqslant \chi_{r-1, \alpha=0.02}^{2}=15.16823
$$

This allows us to say that these data can correspond appropriately to the ZTPBrXW model with $\alpha=0.02$ as a critical value. 


\subsubsection{Example 2}

This data is about the remission times (in months) of a random sample of 128 bladder cancer patients. We choose for this data: $r=11$ intervals, then we obtain the MLE's parameters of our zero truncated Poisson Burr X Lomax distribution as:

$$
\widehat{\lambda}=0.1355, \widehat{\theta}=0.2363, \widehat{\alpha}=0.8491, \widehat{\beta}=1.0072 .
$$

Then the value of $Y_{n}^{2}\left(\widehat{\Theta}_{n}\right)$ is 17.0954. We can see that

$$
Y_{n}^{2}\left(\widehat{\Theta}_{n}\right) \leqslant \chi_{r-1, \alpha=0.05}^{2}=18.30704
$$

It can therefore be concluded that data of remission times of bladder cancer patients is distributed according to our new ZTPBrXLx model.

\section{Concluding remarks}

The goal of this paper is to introduce a new family of continuous distributions with important physical applications. Some statistical properties are derived, certain useful characterizations of the proposed distribution are presented. Five applications are provided to illustrate the importance of the new family. A modified Goodness-of- fit test for the new family in complete data case are investigated via two examples. We propose, as a first step, the construction of Nikulin-Rao-Robson statistic based on chi-squared fit tests for the new family in the case of complete data. The new test is based on the Nikulin-Rao-Robson statistic separately proposed by Nikulin [29] and Rao and Robson [31]. As a second step, an application to real data has been considered to show the maneuverability of the proposed test.

\section{References}

[1] T. H. M. Abouelmagd, A new flexible version of the Lomax distribution with applications, Int. J. Stat. Probab., 7 (2018), 120-132. 7, 7.2

[2] A. Z. Afify, G. M. Cordeiro, H. M. Yousof, A. Alzaatreh, Z. M. Nofal, The Kumaraswamy transmuted-G family of distributions: properties and applications, J. Data Sci., 14 (2016), 245-270. 6.1

[3] A. Z. Afify, Z. M. Nofal, A. N. Ebraheim, Exponentiated transmuted generalized Rayleigh distribution: A new four parameter Rayleigh distribution, Pak. J. Stat. Oper. Res., 11 (2015), 115-134. 6.2

[4] A. Z. Afify, H. M. Yousof, G. M. Cordeiro, E. M. M. Ortega, Z. M. Nofal, The Weibull Fréchet distribution and its applications, J. Appl. Statist., 43 (2016), 2608-2626. 6.1

[5] G. R. Aryal, H. M. Yousof, The exponentiated generalized-G Poisson family of distributions, Stoch. Qual. Control, 32 (2017), 7-23.

[6] V. Bagdonavicius, J. Kruopis, M. S. Nikulin, Non-parametric tests for complete data, John Wiley \& Sons, Hoboken, (2011). 8

[7] V. Bagdonavicius, R. Levuliené, M. S. Nikulin, Q. X. Tran, On chi-squared type tests and their applications in survival analysis and reliability, Zap. Nauchn. Sem. S.-Peterburg. Otdel. Mat. Inst. Steklov. (POMI), 408 (2012), 43-61. 8

[8] E. Brito, G. M. Cordeiro, H. M. Yousof, M. Alizadeh, G. O. Silva, Topp-Leone Odd Log-Logistic Family of Distributions, J. Stat. Comput. Simul., 87 (2017), 3040-3058. 1

[9] G. M. Cordeiro, A. Z. Afify, H. M. Yousof, R. R. Pescim, G. R. Aryal, The exponentiated Weibull-H family of distributions: Theory \& Applications, Mediterr. J. Math., 14 (2017), 22 pages. 1

[10] G. M. Cordeiro, E. M. Hashimoto, E. M. M. Ortega, The McDonald Weibull model, Statistics, 48 (2014), $256-278.6 .1$

[11] G. M. Cordeiro, E. M. M. Ortega, S. Nadarajah, The Kumaraswamy Weibull distribution with application to failure data, J. Franklin Inst., 347 (2010), 1399-1429. 6.1

[12] G. M. Cordeiro, E. M. M. Ortega, B. V. Popovic, The Gamma-Lomax distribution, J. Stat. Comput. Simul., 85 (2015), 305-319. 7

[13] G. M. Cordeiro, H. M. Yousof, T. G. Ramires, E. M. M. Ortega, The Burr XII system of densities: properties, regression model and applications, J. Stat. Comput. Simul., 88 (2018), 432-456. 1

[14] F. Drost, Asymptotics for generalized chi-squared goodness-of-fit tests, CWI Tracs, Centre for Mathematics and Computer Sciences, Amsterdam, (1988). 8.1 
[15] W. Glänzel, A characterization theorem based on truncated moments and its application to some distribution families, in: Mathematical Statistics and Probability Theory, 1986 (1986), 75-84. 4

[16] W. Glänzel, Some consequences of a characterization theorem based on truncated moments, Statistics, 21 (1990), 613-618.

[17] R. C. Gupta, P. L. Gupta, R. D. Gupta, Modeling failure time data by Lehman alternatives, Comm. Statist. Theory Methods, 27 (1998), 887-904. 7

[18] G. G. Hamedani, H. M. Yousof, M. Rasekhi, M. Alizadeh, S. M. Najibi, Type I general exponential class of distributions, Pak. J. Stat. Oper. Res., 14 (2018), 39-55. 1

[19] G. G. Hamedani, M. Rasekhi, S. M. Najibi, H. M. Yousof, M. Alizadeh, Type II general exponential class of distributions, Pak. J. Stat. Oper. Res., (2018), forthcoming. 1

[20] C. S. Kakade, D. T. Shirke, Tolerance interval for exponentiated exponential distribution based on grouped data, Int. J. Agric. Stat. Sci., 3 (2007), 625-631.

[21] M. N. Khan, The modified beta Weibull distribution, Hacet. J. Math. Stat., 44 (2015), 1553-1568. 6.1

[22] M. S. Khan, R. King, Transmuted modified Weibull distribution: a generalization of the modified Weibull probability distribution, Eur. J. Pure Appl. Math., 6 (2013), 66-88. 6.1

[23] M. Ç. Korkmaz, A. I. Genç, Two-Sided Generalized Exponential Distribution, Comm. Statist. Theory Methods, 44 (2015), 5049-5070. 1

[24] C. Lee, F. Famoye, O. Olumolade, Beta-Weibull distribution: some properties and applications to censored data, J. Modern Appl. Statist. Methods, 6 (2007), 173-186. 6.1

[25] A. J. Lemonte, G. M. Cordeiro, An extended Lomax distribution, Statistics, 47 (2013), 800-816. 7

[26] K. S. Lomax, Business failures: Another example of the analysis of failure data, J. Amer. Statist. Assoc., 49 (1954), 847-852. 7

[27] F. Merovci, M. Alizadeh, H. M. Yousof, G. G. Hamedani, The exponentiated transmuted-G family of distributions: theory and applications, Comm. Statist. Theory Methods, 46 (2017), 10800-10822. 1

[28] D. N. P. Murthy, M. Xie, R. Jiang, Weibull Models, Wiley-Interscience [John Wiley \& Sons], Hoboken, (2004). 6.1, $7.2,8.2,8.2 .1$

[29] M. S. Nikulin, Chi-square test for continuous distribution with shift and scale parameters, Theory Probab. Appl., 18 (1974), 559-568. 8, 8.1, 9

[30] S. B. Provost, A. Saboor, M. Ahmad, The Gamma-Weibull distribution, Pakistan J. Statist., 27 (2011), 111-131. 6.1

[31] K. C. Rao, B. S. Robson, A chi-squabe statistic for goodies-of-fit tests within the exponential family, Comm. Statist., 3 (1974), 1139-1153. 8, 8.1, 9

[32] S. Rezaei, S. Nadarajah, N. Tahghighnia, New three-parameter lifetime distribution, Statistics, 47 (2013), 835-860. 6.3

[33] M. M. Ristić, N. Balakrishnan, The Gamma-exponentiated exponential distribution, J. Stat. Comput. Simul., 82 (2012), 1191-1206. 6.3

[34] M. H. Tahir, G. M. Cordeiro, M. Mansoor, M. Zubair, The Weibull-Lomax distribution: properties and applications, Hacet. J. Math. Stat., 44 (2015), 455-474. 7.2

[35] A. W. van der Vaart, Asymptotic Statistics, Cambridge University Press, Cambridge, (1998). 8.1

[36] V. Voinov, R. Alloyarova, N. Pya, Recent achievements in modified chi-squared goodness-of-fit testing, in: Statistical Models and Methods for Biomedical and Technical Systems, 2008 (2008), 241-258. 8.1

[37] H. M. Yousof, A. Z. Afify, M. Alizadeh, N. S. Butt, G. G. Hamedani, M. M. Ali, The transmuted exponentiated generalized-G family of distributions, Pak. J. Stat. Oper. Res., 11 (2015), 441-464. 6.1

[38] H. M. Yousof, A. Z. Afify, G. G. Hamedani, G. Aryal, The Burr X generator of distributions for lifetime data, J. Stat. Theory Appl., 16 (2017), 288-305. 1, 7

[39] H. M. Yousof, M. Alizadeh, S. M. A. Jahanshahiand, T. G. Ramires, I. Ghosh, G. G. Hamedani, The transmuted Topp-Leone G family of distributions: theory, characterizations and applications, J. Data Sci., 15 (2017), 723-740.

[40] H. M. Yousof, E. Altun, T. G. Ramires, M. Alizadeh, M. Rasekhi, A new family of distributions with properties, regression models and applications, J. Statist. Manag. Syst., 21 (2018), 163-188.

[41] H. M. Yousof, M. Rasekhi, A. Z. Afify, I. Ghosh, M. Alizadeh, G. G. Hamedani, The beta Weibull-G family of distributions: theory, characterizations and applications, Pakistan J. Statist., 33 (2017), 95-116. 1

\section{Appendix A}

Theorem .1. Let $(\Omega, \mathcal{F}, \zeta)$ be a given probability space and let $\mathrm{H}=[\mathrm{a}, \mathrm{b}]$ be an interval for some $\mathrm{d}<\mathrm{b}$ $(\mathrm{a}=-\infty, \mathrm{b}=\infty$ might as well be allowed $)$. Let $\mathrm{X}: \Omega \rightarrow \mathrm{H}$ be a continuous random variable with the distribution function $\mathrm{F}$ and let $\mathrm{q}_{1}$ and $\mathrm{q}_{2}$ be two real functions defined on $\mathrm{H}$ such that

$$
\mathbf{E}\left[\mathbf{q}_{2}(X) \mid X \geqslant x\right]=\mathbf{E}\left[\mathbf{q}_{1}(X) \mid X \geqslant x\right] \eta(x), \quad x \in H,
$$

is defined with some real function $\eta$. Assume that $\mathrm{q}_{1}, \mathrm{q}_{2} \in \mathrm{C}^{1}(\mathrm{H}), \xi \in \mathrm{C}^{2}(\mathrm{H})$ and $\mathrm{F}$ is twice continuously differentiable and strictly monotone function on the set $\mathrm{H}$. Finally, assume that the equation $\eta \mathrm{q}_{1}=\mathrm{q}_{2}$ has no real 
solution in the interior of $\mathrm{H}$. Then $\mathrm{F}$ is uniquely determined by the functions $\mathrm{q}_{1}, \mathrm{q}_{2}$ and $\eta$, particularly

$$
F(x)=\int_{a}^{x} C\left|\frac{\eta^{\prime}(u)}{\eta(u) q_{1}(u)-q_{2}(u)}\right| \exp (-s(u)) d u,
$$

where the function $\mathrm{s}$ is a solution of the differential equation $\mathrm{s}^{\prime}=\frac{\eta^{\prime} \mathrm{q}_{1}}{\eta \mathrm{q}_{1}-\mathrm{q}_{2}}$ and $\mathrm{C}$ is the normalization constant, such that $\int_{\mathrm{H}} \mathrm{dF}=1$.

We like to mention that this kind of characterization based on the ratio of truncated moments is stable in the sense of weak convergence, in particular, let us assume that there is a sequence $\left\{X_{n}\right\}$ of random variables with distribution functions $\left\{F_{n}\right\}$ such that the functions $q_{1 n}, q_{2 n}$ and $\eta_{n}(n \in \mathbb{N})$ satisfy the conditions of Theorem .1 and let $q_{1 n} \rightarrow q_{1}, q_{2 n} \rightarrow q_{2}$ for some continuously differentiable real functions $\mathrm{q}_{1}$ and $\mathrm{q}_{2}$. Let, finally, $X$ be a random variable with distribution $F$. Under the condition that $q_{1 n}(X)$ and $q_{2 n}(X)$ are uniformly integrable and the family $\left\{F_{n}\right\}$ is relatively compact, the sequence $X_{n}$ converges to $X$ in distribution if and only if $\eta_{n}$ converges to $\eta$, where

$$
\eta(x)=\frac{\mathbf{E}\left[\mathbf{q}_{2}(X) \mid X \geqslant x\right]}{\mathbf{E}\left[\mathbf{q}_{1}(X) \mid X \geqslant x\right]}
$$

This stability theorem makes sure that the convergence of distribution functions is reflected by corresponding convergence of the functions $\mathrm{q}_{1}, \mathrm{q}_{2}$, and $\eta$, respectively. It guarantees, for instance, the 'convergence' of characterization of the Wald distribution to that of the Lévy-Smirnov distribution if $\alpha \rightarrow \infty$. A further consequence of the stability property of Theorem .1 is the application of this theorem to special tasks in statistical practice such as the estimation of the parameters of discrete distributions. For such purpose, the functions $q_{1}, q_{2}$, and, specially, $\eta$ should be as simple as possible. Since the function triplet is not uniquely determined it is often possible to choose $\eta$ as a linear function. Therefore, it is worth analyzing some special cases which helps to find new characterizations reflecting the relationship between individual continuous univariate distributions and appropriate in other areas of statistics. 